\title{
Fast Transport of RNA Granules by Direct Interactions with KIF5A/KLC1 Motors Prevents Axon Degeneration
}

Yusuke Fukuda ${ }^{1,2}$, Maria F. Pazyra-Murphy ${ }^{1,2}$, Ozge E. Tasdemir-Yilmaz ${ }^{1,2}$, Yihang Li ${ }^{1,2}$, Lillian Rose ${ }^{1,2}$, Zoe C. Yeoh', Nicholas E. Vangos' ${ }^{2}$, Ezekiel A. Geffken ${ }^{2}$, Hyuk-Soo Seo ${ }^{2,3}$, Guillaume Adelmant ${ }^{2,4}$, Gregory H. Bird $^{2,5,6}$, Loren D. Walensky ${ }^{2,5,6}$ Jarrod A. Marto ${ }^{2,4}$, Sirano Dhe-Paganon ${ }^{2,3}$, and Rosalind A. Segal ${ }^{1,2,7, *}$

${ }^{1}$ Department of Neurobiology, Harvard Medical School, Boston, MA 02115, USA

${ }^{2}$ Department of Cancer Biology, Dana-Farber Cancer Institute, Boston, MA 02115, USA

$10{ }^{3}$ Department of Biological Chemistry \& Molecular Pharmacology, Harvard Medical School, Boston, MA

1102115 , USA

12 'Blais Proteomics Center, Dana-Farber Cancer Institute, Boston, MA 02115, USA

135 Linde Program in Cancer Chemical Biology, Dana-Farber Cancer Institute, Boston, MA 02115, USA

$14{ }^{6}$ Department of Pediatric Oncology, Dana-Farber Cancer Institute, Boston, MA 02115, USA

$15{ }^{7}$ Lead Contact

16 *Correspondence: rosalind_segal@dfci.harvard.edu (R.A.S.)

\section{Abstract}

21 Complex neural circuitry requires stable connections formed by lengthy axons. To maintain these functional circuits, fast transport delivers RNAs to distal axons where they undergo local translation.

23 However, the mechanism that enables long distance transport of non-membrane enclosed organelles

24 such as RNA granules is not known. Here we demonstrate that a complex containing RNA and the RNA-

25 binding protein (RBP) SFPQ interacts directly with a tetrameric kinesin containing the adaptor KLC1 and

26 the motor KIF5A. We show that binding of SFPQ to KIF5A/KLC1 motor complex is required for axon survival

27 and is impacted by KIF5A mutations that cause Charcot-Marie-Tooth (CMT) Disease. Moreover,

28 therapeutic approaches that bypass the need for local translation of SFPQ-bound proteins prevent axon

29 degeneration in CMT models. Collectively, these observations show that non-membrane enclosed

30 organelles can move autonomously and that replacing axonally translated proteins provides a therapeutic

31 approach to axonal degenerative disorders. 


\section{Introduction}

Sensory and motor neurons transmit signals through axons than can exceed a meter in length.

34 Therefore, many axonal functions, including axonal survival pathways, depend on proteins that are locally

35 translated and replenished in axon terminals. Localized protein synthesis is enabled by the initial assembly

36 of mRNAs and RNA-binding proteins (RBPs) into ribonucleoprotein (RNP) granules which occurs within the

37 cell soma, transport of these RNA granules to axon endings, and subsequent release of RNA for local

38 protein synthesis (Das, Singer, \& Yoon, 2019). Human mutations that disrupt RNA granule formation,

39 interfere with cytoskeletal structures, or alter activity of intracellular motors and thus interfere with

40 granule transport are a major cause of neurologic diseases including amyotrophic lateral sclerosis (ALS),

41 hereditary spastic paraplegia (HSP) and Charcot-Marie-Tooth (CMT) disease. Degeneration of axons

42 occurs early in such neurodegenerative disorders and precedes cell death of the affected neurons.

43 While transport of RNA granules to axons is an important step in the homeostasis of RNA and

44 proteins in axons, the mechanism by which these non-membrane enclosed organelles are transported by

45 microtubule-dependent motors is not yet understood. A recent study revealed one mechanism for long-

46 range axonal transport in which actin-containing RNA granules “hitchhike" on lysosomes (Liao et al., 2019).

47 However, it is not known if this represents a uniform mechanism for transport of diverse RNA-granules,

48 or whether some types of RNA granules can be transported by motors independently of membrane

49 containing structures.

Splicing factor proline/glutamine-rich (SFPQ) is a ubiquitous RBP that has critical functions in

51 axons of both sensory and motor neurons (Cosker, Fenstermacher, Pazyra-Murphy, Elliott, \& Segal, 2016;

52 Pease-Raissi et al., 2017; Thomas-Jinu et al., 2017). In sensory neurons, SFPQ assembles neurotrophin-

53 regulated transcripts, such as bc/w and Imnb2, to form RNA granules, and is required for axonal

54 localization of these mRNAs and their subsequent translation (Cosker et al., 2016; Pease-Raissi et al.,

55 2017). Similar to many other RBPs, SFPQ contains an intrinsically disordered region (Introduction-figure 
supplement 1) and has been demonstrated to be a component of large RNA transport granules in neurons

57 (Kanai, Dohmae, \& Hirokawa, 2004). Thus, loss of SFPQ leads to depletion of axonal mRNAs and results in

58 axon degeneration in dorsal root ganglion (DRG) sensory neurons (Cosker et al., 2016). Similarly, SFPQ is

59 critical for the development and maintenance of motor neuron axons (Thomas-Jinu et al., 2017). Missense

60 mutations in the coiled coil region of SFPQ have been identified that cause familial ALS and impair the

61 localization of SFPQ within distal axon segments (Thomas-Jinu et al., 2017). However, we do not yet

62 understand the mechanisms by which this RBP organizes mRNA transport granules that can then move

63 rapidly to distal axons where the mRNA cargos are released and translated.

Three distinct, but closely related genes, KIF5A, KIF5B and KIF5C, encode the conventional kinesin-

651 family of motor heavy chains, which are required for anterograde axonal transport of diverse organelles.

66 Mutations in KIF5A cause axonal degenerative disorders including CMT Type 2D (CMT2D), HSP and ALS

67 (Millecamps \& Julien, 2013; Sleigh, Rossor, Fellows, Tosolini, \& Schiavo, 2019). It has been proposed that

KIF5A mutations may cause neurologic diseases by affecting transport efficiency overall. However, mutations in KIF5B or KIF5C do not cause similar neurologic disorders, suggesting that mutations in KIF5A

70 may instead initiate disease due to impaired transport of KIF5A-specific cargo(s). Here, we show that the

71 degeneration of axons in KIF5A or SFPQ-mutant neurons reflects failure to transport a specific non-

72 membrane enclosed organelle rather than a general loss of transport. We identify this cargo as SFPQ-RNA

73 granules and show that a stable small peptide that mimics the function of a locally translated protein can

rescue degeneration caused by defective axonal transport. 


\section{Results}

\section{7}

SFPQ granule, a non-membrane enclosed organelle, undergoes fast axonal transport. mechanisms by which SFPQ and its critical RNA cargos are transported between these two locations is not known. We utilized live cell imaging of DRG sensory neurons expressing Halo-tagged SFPQ to directly visualize transport dynamics (Video 1). Fluorescent signal was enriched in the nucleus and was also evident as discrete granules in the soma and axons, a pattern similar to the distribution of endogenous SFPQ (Cosker et al., 2016). Consistent with the presence of intrinsically disordered regions within the SFPQ coding sequence, Halo-tagged SFPQ granules exhibited liquid like properties during time-lapse imaging (Gopal, Nirschl, Klinman, \& Holzbaur, 2017), as the size and shape of SFPQ granules remained constant at approximately $1 \mu \mathrm{m}$ in diameter during the stationary phase, but the granules expanded and elongated as they move (Figure 1A and 1B). The majority of the Halo-tagged SFPQ granules in axons were motile, either moving by retrograde transport ( $48 \%$ ), or anterograde transport ( $28 \%$ ), with the remainder in stationary phase ( 25\%) (Figure 1C and Figure 1-figure supplement 1A). SFPQ granules exhibit an average anterograde velocity of $0.89 \pm 0.08 \mu \mathrm{m} / \mathrm{sec}$ and average anterograde cumulative displacement of $21.02 \pm 2.49 \mu \mathrm{m}$, with an average retrograde velocity of $0.80 \pm 0.04 \mu \mathrm{m} / \mathrm{sec}$ retrograde and average retrograde cumulative displacement of $32.02 \pm 2.45 \mu \mathrm{m}$ (Figure 1D, Figure 1-figure supplement 1B-E). Together, the velocity and the characteristics of movement indicate that the SFPQgranules are non-membrane enclosed organelles that move in both directions by microtubuledependent fast axonal transport, using a kinesin motor for anterograde and the more highly processive dynein motor for retrograde movements. 
SFPQ preferentially binds to KIF5A/KLC1 motor complex.

The anterograde kinesins involved in axon transport are formed by two dimers of kinesin heavy

three distinct genes, KIF5A, KIF5B and KIF5C, and the genome also contains several light chains, KLC1-4.

104 To identify motors that associate with SFPQ and might enable transport of these RNA granules, we took

105 an unbiased approach in which we immunoprecipitated endogenous SFPQ from DRG neurons and used

106 mass spectrometry to analyze the co-precipitated components. We detected known interactors, including

107 the Drosophila behavior/human splicing (DBHS) protein family members NONO and PSPC1 in the

108 precipitated proteins (Supplementary File 1). We then analyzed the relative abundance of KIF5A, KIF5B

109 and KIF5C (Figure 2B) and KLC1, KLC2 (Figure 2C) in the SFPQ immunoprecipitates using a targeted mass

110 spectrometry approach. We found that KIF5A and KLC1 were each highly enriched over the other proteins

111 (KIF5B or KIF5C and KLC2, respectively) as measured across three independent experiments (Figure 2B,

112 2C and Figure 2-figure supplement 1A). In contrast, when we purified endogenous KLC1 from DRG

113 neurons and analyzed the composition of the resulting immunoprecipitate by mass spectrometry, we

114 observed that all three KIF5 proteins were present at approximately the same abundance in KLC1

115 immunoprecipitate (Figure 2-figure supplement 1A and 1B). Similarly, validated antibodies specific to

116 each of the three KIF5s (Figure 2-figure supplement 1C and 1D) corroborate that SFPQ preferentially co-

117 immunoprecipitates with KIF5A, rather than the closely related KIF5B or KIF5C (Figure 2D and 2E), and

118 with KLC1 rather than KLC2 (Figure 2F). Together these results suggest the possibility that KIF5A/KLC1

119 tetramers may be the distinctive motors responsible for rapid anterograde transport of SFPQ-RNA 120 granules.

121 A previous study of kinesin-1 motors demonstrates that overexpressed KIF5A, B and C can all

122 traffic to axons, but KIF5A is excluded from dendrites (Lipka, Kapitein, Jaworski, \& Hoogenraad, 2016).

123 DRG sensory neurons are pseudo-unipolar in morphology and so have no dendrites, but instead consist 
124 of a cell body with a T-shaped axon. To determine whether the intracellular distribution of kinesins is

125 consistent with the hypothesis that KIF5A/KLC1 is the motor that transports SFPQ-RNA granules, we

126 cultured DRG sensory neurons in compartmented cultures and collected protein lysates distinctly from

127 cell body (CB) and distal axon (DA) compartments. While KIF5B preferentially localizes in the CB

128 compartment, KIF5A and KIF5C localize to both CB and DA compartments, as do KLC1 and KLC2 (Figure

129 2G). Immunostaining of KIF5s in DRG neurons cultured in microfluidic chambers as well as DRGs and sciatic

130 nerves in vivo displayed a similar localization pattern, with KIF5B largely excluded from axons (Figure $\mathbf{2 H}$

131 and Figure 2-figure supplement 2). Together these data demonstrate that KIF5A and KLC1 are 132 appropriately localized to mediate transport of SFPQ-RNA granules from cell bodies to distal axons, and

133 thus KIF5A/KLC1 may represent a specialized motor for these non-membrane enclosed organelles.

RNase prevents SFPQ-RNA binding to KIF5A/KLC1.

As RNA is a critical component of the large SFPQ-containing granules that move rapidly within the

137 axons, we asked whether SFPQ that binds to KIF5A/KLC1 is also associated with RNA cargos. We expressed

138 HA-SFPQ, FLAG-KIF5A and KLC1-Myc in HEK293T cells, and treated the cell lysates with RNase, or vehicle

139 control, and then immunoprecipitated for HA-SFPQ. Strikingly we find that RNase treatment impeded the

140 interaction between SFPQ and KIF5A-KLC1 (Figure 3A-C), demonstrating that SFPQ only binds KIF5A/KLC1

141 when it is associated with RNA. These findings suggest that KIF5A/KLC1 bind and transport SFPQ when it

142 is part of a large RNP transport granule.

143 Based on the above findings, we hypothesize that KIF5A/KLC1 distinctively mediates fast transport

144 of critical SFPQ-RNA granules from the cell soma to the axons. To identify the structural basis for this

145 specificity, we first asked whether the highly divergent C-terminal tail regions of KLC1 and KIF5A are

146 required for SFPQ binding. When we overexpressed either Myc-tagged WT KLC1 or its C-terminal mutant

147 ( $\triangle$ Tail) in HEK 293T cells, and assessed binding to SFPQ by co-precipitation studies, we find that the binding 
148 is reduced by approximately $50 \%$ in the absence of the C-terminal region of KLC1 (Figure 3D-F). Similarly,

149 truncation of the highly variable tail region of KIF5A nearly abolished its interaction with SFPQ (Figure 3G-

150 I) although this did not prevent binding of KIF5A to KLC1 (Figure 3-figure supplement 1). Together these

151 data demonstrate that SFPQ binding to KIF5A/KLC1 is enabled by the highly divergent C-terminal regions

152 of both KLC1 and KIF5A.

SFPQ directly binds to KLC1 through a Y-acidic motif within its coiled coil domain.

Evidence that SFPQ binding to KIF5A/KLC1 requires concurrent binding to RNA, suggests that this

RBP may directly link RNA transport granules to kinesin motors, enabling these RNA granules to move

157 autonomously without a membranous platform. To develop novel tools for testing this possibility, we

158 sought to identify specific mutations in SFPQ that impair binding to kinesin. SFPQ contains a Y-acidic motif

159 that is evolutionary conserved and closely matches the Y-acidic sequence of JIP1 that connects

160 membranous organelles to KLC1 (Nguyen et al., 2018; Pernigo et al., 2018) (Figure 4A and Figure 4-figure

161 supplement 1). When we mutated the critical tyrosine residue within the motif to alanine (Y527A) the

162 binding between SFPQ and KIF5A/KLC1 was dramatically reduced, demonstrating that this Y-acidic motif

163 is required for binding to KIF5A/KLC1 motor complex (Figure 4B and 4C). To assess whether SFPQ binds

164 directly to KLC1 without requiring a membranous organelle or an adaptor component, we purified human

165 KLC1 and used isothermal titration calorimetry (ITC) to test direct binding by a long SFPQ peptide that

166 spans the Y-acidic motif. The SFPQ peptide binds directly to KLC1 with a Kd of $3.8 \pm 2.3 \mu \mathrm{M}$ and a binding

167 stoichiometry of 1 (Figure 4D and Table 1). Consistent with data from co-immunoprecipitation studies

168 above, Y527A mutation prevents the SFPQ peptide from binding directly to KLC1 in ITC assays (Figure 4D).

169 Thermodynamic parameters of ITC measurements are summarized in Table 1 . Together these data

170 demonstrate that SFPQ directly binds to KLC1 in a process that relies on the Y-acidic motif and is abrogated

171 by the Y527A mutation. These studies suggest that SFPQ RNA-transport granules might directly associate 
172 with microtubule-dependent motors rather than requiring a membrane platform for intracellular

173 transport.

175 Direct binding of SFPQ to KIF5A/KLC1 is required for its transport in axons.

The Y527A mutant of SFPQ provides a tool that can be used to ask whether the direct interaction

182 SFPQ within DRG neurons grown in microfluidic chambers. In neurons expressing the Y527A mutant

183 (Video 2), the number of SFPQ particles localized to distal axons was reduced by 50\%, suggesting that

184 direct binding of SFPQ to KIF5A/KLC1 is required for the redistribution of SFPQ-granules from the cell

185 bodies to distal axons (Figure 5A and 5B). Moreover, among the SFPQ granules that reached the axons,

186 the Y527A mutant SFPQ exhibited a $~ 50 \%$ reduction in the percentage of time spent in anterograde axonal

187 transport compared to WT (Figure 5C). Since SFPQ forms a dimer, residual movement of Y527A may

188 reflect binding of KIF5A/KLC1 motors to dimeric SFPQ containing both endogenous WT SFPQ and the

189 fluorescent mutant isoform (Hewage, Caria, \& Lee, 2019). Together these data demonstrate that defects

190 in the direct binding of Y527A to KIF5A/KLC1 motor complex interrupts anterograde transport of RNA

191 granules in axons of DRG sensory neurons, suggesting that these RNA granules move autonomously and

192 do not require a membrane platform.

193 It is striking that the Y-acidic motifs in JIP1 and other proteins that link kinesins to membranous

194 organelles are usually located within highly accessible regions such as the carboxy terminus, while the Y-

195 acidic motif in SFPQ is instead located within the highly structured coiled-coil domain. These structural 
196 differences suggest that SFPQ-RNA granules may interact with kinesin in a different manner than do

197 membranous organelles. Previous studies identified sequences within KLC1 that are not present in KLC2

198 and that specify binding to JIP1. One key residue in KLC1 is N343 within the TPR4 region of KLC1; mutation

199 of this residue to a serine, as observed in KLC2, abrogates interaction between JIP1 and KLC1 (Zhu et al.,

200 2012) (Figure 5-figure supplement 1A). To determine whether N343 on KLC1 also mediates binding to

201 SFPQ, we expressed myc-tagged WT or N343S KLC1 together with HA-tagged SFPQ in HEK 293T cells. We

202 find that KLC1-N343S did not alter binding to SFPQ, in clear contrast to JIP1 (Figure 5-figure supplement

203 1B), suggesting that the way in which SFPQ-RNA granules bind to kinesin motors for transport differs from

204 that observed with JIP1.

Defect in KIF5A-driven transport of SFPQ leads to axon degeneration in DRG sensory neurons.

To determine the physiologic consequences of impeding SFPQ transport by KIF5A/KLC1, we

leveraged the SFPQ Y527A mutant (Figure 6A and 5). As shown previously, knockdown of SFPQ results in axon degeneration . We then assessed the ability of constructs encoding either the WT SFPQ or SFPQ-

210 Y527A to reverse the degeneration caused by knockdown, using constructs resistant to shRNA knockdown

211 (Figure 6B and Figure 6-figure supplement 1). Strikingly, expression of the WT version rescued axon

212 degeneration caused by knockdown of SFPQ, whereas expression of SFPQ Y527A was unable to do so, and

213 instead led to the same degree of axon degeneration observed following knockdown of SFPQ (Figure 6C

214 and 6D). Taken together these data indicate that autonomous transport of SFPQ-RNA granules is required

215 for axon survival and demonstrate that defects in kinesin-driven transport of SFPQ causes degeneration

216 of sensory axons.

217 Our data indicate that KIF5A/KLC1 functions as the motor transporting SFPQ-RNA granules within

218 DRG sensory neurons, and that this distinct transport process is required for axon survival. Thus, CMT2D

219 mutations of KIF5A may cause defects in this specialized transport pathway and so compromise axonal 
220 health. Mutations causing HSP and CMT2D are located primarily within the motor domain of KIF5A; each

221 distinct mutation can give rise to HSP or CMT2D or a combination of both syndromes. Two independent

222 studies have reported a R280H mutation that results in a pure classical form of CMT2D (Figure 6A) (Liu et

223 al., 2014; Nam, Yoo, Choi, Choi, \& Chung, 2018). Both structural and biochemical characterization

224 indicates that disease mutations altering this residue reduce the microtubule binding affinity, and

225 therefore decrease transport (Dutta, Diehl, Onuchic, \& Jana, 2018; Ebbing et al., 2008; Fuger et al., 2012;

226 Jennings et al., 2017). Interestingly, despite the mutation residing within the motor domain, R280H

227 mutation also reduced binding to SFPQ approximately 25\% compared to WT KIF5A (Figure 6-figure

228 supplement $2 \mathrm{~A}$ and $2 \mathrm{~B}$ ). As $\mathrm{CMT} 2 \mathrm{D}$ mutation within $\mathrm{R} 280 \mathrm{H}$ not only reduces microtubule binding affinity

229 and transport but also impacts binding to SFPQ, this pathologic mutation is likely to disproportionately

230 affect transport of the RNA cargo transported together with SFPQ.

231 To model axon degeneration induced by KIF5A mutation in R280H in vitro, we expressed the KIF5A

232 R280H mutation by lentivirus in DRG sensory neurons that also express the endogenous WT KIF5A. As

233 observed in patients heterozygous for CMT2D mutations, overexpression of $\mathrm{R} 280 \mathrm{H}$ led to axon

234 degeneration in DRG sensory neurons (Figure 6E and 6F), while control studies indicate that

235 overexpression of WT does not compromise axon integrity (Figure 6E and 6F). This model provides a

236 platform for investigating the molecular changes that cause degeneration in CMT disease.

238 Axon degeneration caused by CMT2D R280H KIF5A mutation can be rescued by a Bclw mimetic peptide.

240 organelles as well as RNA granules; thus degeneration of axons caused by R280H KIF5A mutation could

241 be a consequence of defect in transport of any or all of these cargos. A similar pattern of axon

242 degeneration is observed in sensory neurons expressing the SFPQ Y527A mutant, suggesting that SFPQ-

243 RNA granules may represent a critical cargo impacted in CMT patients with KIF5A R280H mutations. Local 
244 translation of mRNAs bound to SFPQ is a critical step that promotes axon survival; $b c / w$ is one such mRNA

245 that is bound by SFPQ and is translated in axons of DRG sensory neurons (Figure 7A). We asked whether

246 restoring functions downstream of the SFPQ pathway can rescue degeneration caused by KIF5A R280H

247 mutant. Intriguingly, a BH4 peptide mimetic of Bclw introduced into axons of R280H KIF5A mutant can

248 prevent axon degeneration in this genetic model of CMT with axonal survival returning to the levels

249 observed in control or WT KIF5A overexpressing DRG neurons (Figure 7B). This effect is specific to Bclw

250 since introducing a peptide mimetic of related $\mathrm{Bc} / 2$ proteins did not prevent degeneration, and

251 degeneration remained at the level observed with the $\mathrm{R} 280 \mathrm{H}$ mutant alone (Figure $7 \mathrm{C}$ ). Taken together,

252 these data suggest that defective transport of SFP-RNA granules containing bc/w mRNA represents a key

253 mechanism that underlies CMT2D-causing mutations of KIF5A, and so causes axon degeneration.

\section{Discussion}

Kinesins are a large family of microtubule-dependent motors that play a pivotal role in rapid

257 intracellular transport. These motors are particularly critical in neurons that form extensive axonal and

258 dendritic projections and so rely on fast transport across long distances. While mutations in kinesin motors

259 cause neurodegenerative diseases affecting primary sensory and motor neurons, it is not known whether

260 this reflects a requirement for specific kinesins in mediating transport of particular cargos within the

261 lengthy axons that extend to peripheral targets, or whether degeneration reflects a more global loss of

262 axonal transport. Here we show that the non-membrane enclosed RNA granules containing the RNA

263 binding protein SFPQ selectively and directly interact with kinesin containing the KIF5A heavy chain and

264 the cargo adaptor KLC1. Therefore, mutations in KIF5A that cause sensory neuropathy preferentially

265 impact motility of SFPQ granules that transport $b c / w$ and other mRNAs, and the degeneration caused by

266 KIF5A mutations can be prevented by a peptide that mimics the function of the locally translated protein

267 Bclw. 
Although it is widely accepted that RNA-granules are non-membrane enclosed organelles that

269 move by microtubule-dependent transport, how such RNA-granules associate with motors and move

270 through the axoplasm is not yet known. Our data demonstrate a direct interaction between SFPQ and the

271 kinesin-1 cargo adaptor complex KLC1, and we show that this interaction is required for autonomous

272 axonal transport. In contrast to this direct transport system, a recent study by Liao et al. demonstrated

273 that RNA-granules containing $\beta$-actin mRNA rely on annexin A11 adaptor protein to hitchhike on

274 lysosomes and thereby regulate growth cone morphology (Liao et al., 2019). As SFPQ does not bind $\beta$ -

275 actin but instead binds mRNAs that promote axonal survival, our findings indicate that distinct pools of

276 RNA-granules containing different mRNAs rely on divergent modes of axonal transport.

Data from quantitative mass spectrometry and isothermal titration calorimetry demonstrate that

278 SFPQ preferentially binds KLC1/KIF5A in DRG sensory neurons and that an evolutionarily conserved Y-

279 acidic motif is sufficient for binding directly to KLC1. Interestingly, binding of the protein cargo/adaptors

280 JIP1, TorsinA and SH2D6 to KLC1 require Y-acidic motifs within an unstructured part of the molecule

281 (Nguyen et al., 2018; Pernigo et al., 2018), whereas the motif within SFPQ resides in the highly structured

282 coiled-coil region of SFPQ (Figure 4A and Figure 4-figure supplement 1). As RNA interactions enable SFPQ

283 to bind KIF5A/KLC1, formation of a RNP complex may expose the Y-acidic motif within the coiled-coil

284 domain. Thus, the structural basis for the interaction of KIF5A/KLC1 with SFPQ-RNA granules is likely to

285 differ from the mechanism for binding of KIF5A/KLC1 to JIP1. Consistent with this distinction, the N343S

286 mutation of KLC1 that disrupts interaction with JIP1 (Pernigo et al., 2018; Zhu et al., 2012) has no effect

287 on binding with SFPQ (Figure 5-figure supplement 1B). Instead the highly divergent C-terminal region of

288 KLC1 affects binding to SFPQ (Figure 3D-F). As the carboxy terminal region of KLC1 diverges among several

289 splice variants of KLC1 (McCart, Mahony, \& Rothnagel, 2003), individual isoforms of KLC1 that are present

290 in DRG sensory neurons may specify binding to SFPQ-RNA granules. 
Similar to other phase separating RBPs, SFPQ contains an intrinsically disordered region in its N-

292 terminal region (Introduction-figure supplement 1) and SFPQ assembles into large RNA transport

293 granules, which are non-membrane enclosed organelles (Kanai et al., 2004). In the nucleus, SFPQ interacts

294 with members of the DBHS proteins, NONO and PSPC1, and also binds RNA fragments of NEAT1_2 in order

295 to form paraspeckle nuclear bodies, another phase separating structure (Yamazaki et al., 2018). As is the

296 case for paraspeckle formation, RNA is a critical component of transport granules; RNase treatment leads

297 to loss of integrity of these axonal granules (Knowles et al., 1996), and binding between SFPQ and

298 KIF5A/KLC1 is highly sensitive to RNase treatment (Figure 3A-C). Binding to mRNAs such as bc/w may

299 induce a conformational change in SFPQ that facilitates oligomerization and phase separation and exposes

300 the Y-acidic motif within the coiled coil domain so it can bind KLC1/KIF5A. Further structural studies

301 investigating how specific RNA cargos regulate the conformation and binding of SFPQ to KLC1 and KIF5A

302 will be necessary to fully evaluate this hypothesis.

303 Among the three KIF5 genes encoding the kinesin-1 family of motors, the KIF5A gene is the only

304 one associated with the human neurological diseases CMT2D, HSP and ALS. Our results demonstrate that

305 binding of SFPQ to KLC1 complexed with KIF5A rather than KIF5B or KIF5C enables transport of SFPQ-RNA

306 granules and promotes axon survival. Based on these findings, we postulate that defective transport of

307 SFPQ-RNA granules is a major contributor to KIF5A-associated neurodegenerative disorders, rather than

308 axon degeneration in these disorders being the result of a generalized impairment of transport.

SFPQ RNA-granules are critical for development and maintenance of axons in motor neurons as

310 well as in sensory neurons; and human mutations in SFPQ and KIF5A have been implicated in ALS (Thomas-

311 Jinu et al., 2017). Interestingly, the Y527 residue of the Y-acidic motif lies adjacent to the two identified

312 ALS mutations: N533H and L534I; these two mutations lead to defects in the axonal functions of SFPQ in

313 motor neurons (Thomas-Jinu et al., 2017) and our data suggest that this may reflect altered binding of

314 SFPQ to KIF5A/KLC1 and altered autonomous transport of these non-membrane enclosed granules. 
315 Moreover, ALS-associated mutations in KIF5A transform the C-terminal region of the protein (Nicolas et

316 al., 2018), the domain required for SFPQ binding. Therefore, altered binding and axonal transport of SFPQ

317 may explain the axon degeneration of motor neurons in patients harboring ALS-associated mutations in

318 SFPQ or KIF5A. A recent study by Luisier et al. demonstrated that aberrant localization of SFPQ is also a

319 molecular hallmark of multiple familial and sporadic models of ALS that do not exhibit mutations in SFPQ

320 or KIF5A (Luisier et al., 2018), suggesting that disrupted transport and axonal function of SFPQ may

321 contribute to additional neurodegenerative disorders. Here we demonstrated that a Bclw peptide

322 mimetic rescues axon degeneration caused by R280H KIF5A mutations (Figure 7), extending previous

323 evidence that this therapeutic approach prevents paclitaxel-induced axon degeneration (Pease-Raissi et

324 al., 2017). Taken together, our data suggests that Bclw peptide mimetics should be explored as a potential

325 therapeutic intervention for preventing axon degeneration in multiple neurological diseases that share

326 defects in RNA transport as part of their pathophysiology.

\section{Acknowledgments}

328 We thank Thomas Schwarz and Himanish Basu (Boston Children's Hospital, MA) for Kymolyzer software.

329 We thank Gary Banker (Oregon Health and Science University, OR) and Marvin Bentley (Rensselaer

330 Polytechnic Institute, NY) for KIF5A, KIF5B, KIF5C and KLC1 constructs; and Corinne Houart (Kings's College

331 London, United Kingdom) for SFPQ construct. We thank the Segal lab, Charles Stiles and Michael

332 Greenberg for helpful comments on this manuscript.

\section{Declaration of Interests}

R.A.S. family member is on BoD for Allergen; SAB member for Amgen and Decibel Therapeutics. L.D.W. is AstraZeneca and serves on the SAB of 908 Devices. 
Table for Reagents and Resources

\begin{tabular}{|c|c|c|}
\hline REAGENT or RESOURCE & SOURCE & IDENTIFIER \\
\hline \multicolumn{3}{|l|}{ Antibodies } \\
\hline rabbit polyclonal anti-KIF5A; WB, IF & Abcam & ab5628 \\
\hline rabbit polyclonal anti-KIF5B; WB, IF & Abcam & ab5629 \\
\hline rabbit polyclonal anti-KIF5C; WB, IF & Abcam & ab5630 \\
\hline mouse monoclonal anti-SFPQ [B92]; IP & Abcam & ab11825 \\
\hline mouse monoclonal anti-SFPQ; WB & Sigma & WH0006421M2 \\
\hline KLC1 antibody [H75]; WB & Santa Cruz & sc-25735 \\
\hline KLC2 antibody; WB & Proteintech & 17668-1-AP \\
\hline Pan-Actin (D18C11) Rabbit mAb; WB & Cell Signaling & $8456 S$ \\
\hline Normal Mouse IgG; IP & Millipore Sigma & $12-371$ \\
\hline Normal Rabbit IgG; IP & Cell Signaling & $2729 S$ \\
\hline c-Myc Antibody (9E10); WB & Santa Cruz & sc-40 \\
\hline Anti-Myc Antibody; IP & Millipore Sigma & 06-340-MI \\
\hline Monoclonal ANTI-FLAG M2 antibody (M2); WB & Sigma & F1804 \\
\hline HA Tag Monoclonal Antibody (2-2.2.14); WB and IP & Thermo Fisher & 26183 \\
\hline Anti-GFP, N-terminal antibody, WB & Sigma & G1544 \\
\hline Goat Anti-Rabbit IgG (H+L)-HRP conjugate; WB & Bio-Rad & 1721019 \\
\hline Goat Anti-mouse IgG $(\mathrm{H}+\mathrm{L})-\mathrm{HRP}$ conjugate; WB & Bio-Rad & 1706516 \\
\hline Mouse Purified anti-Tubulin $\beta 3$ (TUBB3) Antibody; IF & Biolegend & 801213 \\
\hline Rabbit Purified anti-Tubulin $\beta 3$ (TUBB3) Antibody; IF & Biolegend & 802001 \\
\hline Goat anti-Mouse IgG $(\mathrm{H}+\mathrm{L})$ Alexa Fluor 488; IF & Invitrogen & A-11001 \\
\hline Goat anti-Rabbit IgG $(\mathrm{H}+\mathrm{L})$ Alexa Fluor 546; IF & Invitrogen & A-11010 \\
\hline Donkey anti-Mouse IgG $(\mathrm{H}+\mathrm{L})$ Alexa Fluor 488 & Invitrogen & A-21202 \\
\hline Donkey anti-Mouse IgG $(\mathrm{H}+\mathrm{L})$ Alexa Fluor 647 & Invitrogen & A-31573 \\
\hline \multicolumn{3}{|l|}{ Bacterial and Virus Strains } \\
\hline shSFPQ & Sigma & TRCN0000102240 \\
\hline shKIF5A & Sigma & TRCN0000415243 \\
\hline shKIF5C & Sigma & TRCN0000090857 \\
\hline \multicolumn{3}{|l|}{ Chemicals, Peptides, and Recombinant Proteins } \\
\hline ESEMEDAYHEHQANLLR (SFPQ WT) & KE Biochem & Custom \\
\hline ESEMEDAAHEHQANLLR (SFPQ Y527A) & KE Biochem & Custom \\
\hline Bclw BH4 SAHB & Loren D. Walensky & $\begin{array}{l}\text { Walensky Laboratory, } \\
\text { Dana-Farber Cancer } \\
\text { Institute }\end{array}$ \\
\hline $\mathrm{Bcl} 2 \mathrm{BH} 4 \mathrm{SAHB}_{\mathrm{A}}$ & Loren D. Walensky & $\begin{array}{l}\text { Walensky Laboratory, } \\
\text { Dana-Farber Cancer } \\
\text { Institute }\end{array}$ \\
\hline Puromycin dihydrochloride & Sigma & P9620 \\
\hline Rnase A/T1 Mix & Thermo Fisher & FEREN0551 \\
\hline FuGENE 6 Transfection Reagent & Promega & E2691 \\
\hline Lipofectamine 2000 Transfection Reagent & Invitrogen & 11668027 \\
\hline In-Fusion HD Cloning Plus & Clontech & 638909 \\
\hline Q5 Site-Directed Mutagenesis Kit & $\begin{array}{l}\text { New England } \\
\text { BioLabs }\end{array}$ & E0554S \\
\hline cOmplete, Mini, EDTA-free protease inhibitor cocktail & Millipore Sigma & 11836170001 \\
\hline
\end{tabular}




\begin{tabular}{|c|c|c|}
\hline Halt Phosphatase inhibitor cocktail & Thermo Scientific & 78420 \\
\hline Amersham ECL western blotting detection system & VWR & $95038-570$ \\
\hline SuperSignal West Dura Extended Duration Substrate & Thermo Scientific & 34076 \\
\hline Dynabeads protein $\mathrm{G}$ & Invitrogen & 10003D \\
\hline Sera-Mag Carboxylate-Modified Magnetic Particles & GE Healthcare & 24152105050250 \\
\hline Neurobasal Medium & Gibco & 21103049 \\
\hline Neurobasal Medium, minus phenol red & Gibco & 12348017 \\
\hline Hibernate E Low Fluorescence & Brain Bits & HELF500 \\
\hline B-27 Supplement, serum free & Gibco & 17504044 \\
\hline GlutaMAX Supplement & Gibco & 35050061 \\
\hline DMEM, high glucose, pyruvate & Gibco & 11995065 \\
\hline Fetal Bovine Serum & Corning cellgro & B003L51 \\
\hline Corning Matrigel GFR Membrane Matrix & Fisher Scientific & CB40230C \\
\hline Laminin Mouse Protein, Natural & Gibco & 23017015 \\
\hline Poly-D-lysine hydrobromide & Sigma & P6407 \\
\hline Recombinant Human $\beta$-NGF & Peprotech & $450-01$ \\
\hline Recombinant Human/Murine/Rat BDNF & Peprotech & $450-02$ \\
\hline tetramethyl Rhodamine (TMR) & Promega & G8251 \\
\hline Chariot protein delivery system & Active Motif & 30025 \\
\hline \multicolumn{3}{|l|}{ Critical Commercial Assays } \\
\hline Amicon Ultra-15 Centrifugal Filter Units & Millipore Sigma & UFC910024 \\
\hline NuPAGE $4-12 \%$ Bis-Tris Protein Gels, $1.0 \mathrm{~mm}, 10$ well & Thermo Fisher & NP0321 \\
\hline $450 \mu \mathrm{m}$ microgroove barrier silicone device & Xona Microfluidics & SND450 \\
\hline $900 \mu \mathrm{m}$ microgroove barrier XonaChips & Xona Microfluidics & XC900 \\
\hline Poly-d-lysine solution optimized for Xona platforms & Xona Microfluidics & XonaPDL \\
\hline Teflon Divider chamber, $20 \mathrm{~mm}$ OD & Tyler Research & CAMP10 \\
\hline \multicolumn{3}{|l|}{ Experimental Models: Cell Lines } \\
\hline Human HEK 293T & ATCC & CRL-3216 \\
\hline \multicolumn{3}{|l|}{ Experimental Models: Organisms/Strains } \\
\hline Sprague Dawley Rat & $\begin{array}{l}\text { Charles River } \\
\text { Laboratory }\end{array}$ & $\mathrm{N} / \mathrm{A}$ \\
\hline \multicolumn{3}{|l|}{ Recombinant DNA } \\
\hline FUGW & $\begin{array}{l}\text { (Lois, Hong, Pease, } \\
\text { Brown, \& Baltimore, } \\
\text { 2002) }\end{array}$ & Addgene \#14883 \\
\hline pLV-EF1a-IRES-Puro & (Hayer et al., 2016) & Addgene \#85132 \\
\hline pMD2.G & Didier Trono & Addgene \#12259 \\
\hline pxPAX2 & Didier Trono & Addgene \#12260 \\
\hline UbC-Halo-SFPQ & This paper & $\mathrm{N} / \mathrm{A}$ \\
\hline pLV-EF1a-GFP-SFPQ-IRES-mCherry & This paper & $\mathrm{N} / \mathrm{A}$ \\
\hline pLV-EF1a-GFP-SFPQ (Y527A)-IRES-mCherry & This paper & $\mathrm{N} / \mathrm{A}$ \\
\hline pLV-EF1a-KIF5A-HA-IRES-Puro & This paper & $\mathrm{N} / \mathrm{A}$ \\
\hline pLV-EF1a-KIF5A(R280H)-HA-IRES-Puro & This paper & $\mathrm{N} / \mathrm{A}$ \\
\hline FLAG-KIF5A & This paper & $\mathrm{N} / \mathrm{A}$ \\
\hline FLAG-KIF5A $\Delta$ tail & This paper & $\mathrm{N} / \mathrm{A}$ \\
\hline FLAG-KIF5A (R280H) & This paper & $\mathrm{N} / \mathrm{A}$ \\
\hline HA-KIF5A & This paper & $\mathrm{N} / \mathrm{A}$ \\
\hline HA-SFPQ & This paper & $\mathrm{N} / \mathrm{A}$ \\
\hline
\end{tabular}




\begin{tabular}{|c|c|c|}
\hline FLAG-SFPQ & This paper & $\mathrm{N} / \mathrm{A}$ \\
\hline FLAG-SFPQ (Y527A) & This paper & $\mathrm{N} / \mathrm{A}$ \\
\hline KLC1-Myc & This paper & $\mathrm{N} / \mathrm{A}$ \\
\hline KLC1-Myc (N343S) & This paper & $\mathrm{N} / \mathrm{A}$ \\
\hline Myc-KLC1 & This paper & $\mathrm{N} / \mathrm{A}$ \\
\hline Myc-KLC1 $\Delta$ tail & This paper & $\mathrm{N} / \mathrm{A}$ \\
\hline hsSFPQ pcS2 & $\begin{array}{l}\text { (Thomas-Jinu et al., } \\
2017 \text { ) }\end{array}$ & $\mathrm{N} / \mathrm{A}$ \\
\hline pBa-GFP-Inkr-mmKIF5a & $\begin{array}{l}\text { Gary Banker and } \\
\text { Marvin Bentley }\end{array}$ & $\mathrm{N} / \mathrm{A}$ \\
\hline pBa-GFP-mmKIF5B & $\begin{array}{l}\text { Gary Banker and } \\
\text { Marvin Bentley }\end{array}$ & $\mathrm{N} / \mathrm{A}$ \\
\hline pBa-mCherry-myc-KIF5C & $\begin{array}{l}\text { Gary Banker and } \\
\text { Marvin Bentley }\end{array}$ & $\mathrm{N} / \mathrm{A}$ \\
\hline pBa-GFP-3myc-mmKLC1a & $\begin{array}{l}\text { Gary Banker and } \\
\text { Marvin Bentley }\end{array}$ & $\mathrm{N} / \mathrm{A}$ \\
\hline pET28MHL-hsKLC1 (205-501) & Cheryl Arrowsmith & Addgene \#26096 \\
\hline \multicolumn{3}{|l|}{ Software and Algorithms } \\
\hline (Fiji is just) ImageJ 2.0.0 & $\mathrm{NIH}$ & http://imagej.nih.gov/ij \\
\hline Prism7 & GraphPad Software & https://graphpad.com/ \\
\hline NIS-Elements Imaging Software & Nikon & $\begin{array}{l}\text { https://www.healthcare. } \\
\text { nikon.com/en/ }\end{array}$ \\
\hline NanoAnalyze Software & TA instruments & $\begin{array}{l}\text { https://www.tainstrumen } \\
\text { ts.com/support/software } \\
\text {-downloads- } \\
\text { support/downloads/ }\end{array}$ \\
\hline Kymolyzer & $\begin{array}{l}\text { (Shlevkov et al., } \\
\text { 2019) }\end{array}$ & $\mathrm{N} / \mathrm{A}$ \\
\hline
\end{tabular}

All experimental procedures were done in accordance with the National Institute of Health guidelines and were approved by the Dana-Farber Cancer Institutional Animal Care and Use Committee.

Animal Use: Time pregnant Sprague-Dawley rats were purchased from Charles River.

DNA and shRNA constructs: Constructs used for HEK 293T IP studies were cloned into pcDNA3.1 vector using PCR-based In-Fusion HD cloning (Clontech). KIF5 (pBa-GFP-Inkr-mmKIF5A; pBa-GFP-mmKIF5B; pBamCherry-myc-KIF5C) and KLC1 (pBa-GFP-3myc-mmKLC1a) constructs were a gift from Dr. Garry Banker and Dr. Marvin Bentley and were used as a template to clone HA, FLAG or Myc-tagged constructs: HAKIF5A, FLAG-KIF5A (WT, $\Delta$ tail, and R280H), KLC1-Myc (WT and N343S) and Myc-KLC1 (WT and $\Delta$ tail). Human version of SFPQ (hsSFPQ pcS2) was a gift from Dr. Corinne Houart and was used to clone HA and FLAG-tagged constructs: HA-SFPQ, and FLAG-SFPQ (WT and Y527A). The R280H KIF5A, N343S KLC1 and Y527A SFPQ mutants were generated by Q5 site-directed mutagenesis (NEB) using manufacturer's instructions. Lentiviral constructs used for compartmented Campenot cultures were cloned into pLVEF1a-IRES-Puro, a gift from Tobias Meyer (Addgene plasmid \#85132), for KIF5A-HA (WT and R280H) or into pLV-EF1a-IRES-mCherry for GFP-SFPQ (WT and Y527A). For live cell imaging of SFPQ, Halo-tagged SFPQ was cloned into FUGW, a gift from David Baltimore (Addgene plasmid \#14883), as a backbone vector. The shRNAs against shSFPQ (TRCN0000102240), KIF5A (TRCN0000415243) and KIF5C (TRCN0000090857) were purchased from Mission. 
DRG sensory neuron culture: DRGs from embryonic day 15 rats of either sex were dissected and trypsinized. For mass cultures, 300,000 cells were plated onto p35 dishes coated with Corning Matrigel GFR Membrane Matrix (Thermo Fisher) diluted in DMEM (Thermo Fisher). Cultures were maintained in Neurobasal (Invitrogen) with 2\% B27 supplement (Invitrogen), 1\% Penicillin/streptomycin, 1\% GlutaMAX (Life Technologies) and $0.08 \%$ glucose at $37^{\circ} \mathrm{C}$ and $7.5 \% \mathrm{CO}_{2}$. DRG neurons were plated with $0.3 \mu \mathrm{M} \mathrm{AraC}$; $100 \mathrm{ng} / \mathrm{mL}$ NGF (Peptrotech) + BDNF (Peptrotech); and on DIV2 neurotrophins were reduced to $10 \mathrm{ng} / \mathrm{mL}$ NGF + BDNF with AraC and maintained until DIV6 for collection and lysis.

For compartmented Campenot chambers, 120,000 cells were plated in the cell body compartment of a Teflon divider (Camp10, Tyler Research) attached to a p35 dish coated with Corning Matrigel GFR Membrane Matrix diluted in DMEM. DRG neurons were initially plated with $0.3 \mu \mathrm{M} \mathrm{AraC} ; 100 \mathrm{ng} / \mathrm{mL}$ NGF + BDNF; and on DIV1 neurotrophins were reduced to $10 \mathrm{ng} / \mathrm{mL} \mathrm{NGF} \mathrm{+} \mathrm{BDNF} \mathrm{with} \mathrm{AraC} \mathrm{only} \mathrm{in} \mathrm{the} \mathrm{cell}$ body compartment. On DIV2 cultures were maintained in the same neurotrophin concentration $(10 \mathrm{ng} / \mathrm{mL}$ for cell body and $100 \mathrm{ng} / \mathrm{mL}$ for distal axons) but without AraC and reduced to $0 \mathrm{ng} / \mathrm{mL} \mathrm{NGF}+\mathrm{BDNF}$ in the cell body compartment and $1 \mathrm{ng} / \mathrm{mL} \mathrm{NGF}+$ BDNF in the distal axon compartment with AraC from DIV 5 to DIV8 for collecting protein lysates or for degeneration assay. As efficient knockdown of SFPQ with shRNA takes up to DIV8, the Campenot cultures used for degeneration assay involving shSFPQ were maintained until DIV12 with the following modifications: from DIV5 to DIV8, neurotrophins were maintained at $10 \mathrm{ng} / \mathrm{mL} \mathrm{NGF}+\mathrm{BDNF}$ for both cell body and axons with AraC and then reduced to $1 \mathrm{ng} / \mathrm{mL} \mathrm{NGF}+\mathrm{BDNF}$ for both cell body and axons with AraC until DIV12.

Microfluidic device with $900 \mu \mathrm{m}$ microgroove barrier XonaChip (XC900; Xona) was prepared for live cell imaging following manufacturer's instructions except following the PBS washes for XonaPDL (Xona) coating, the device was further incubated with $10 \mu \mathrm{g} / \mathrm{mL}$ of laminin (Life Technologies) for 3 hours at $37^{\circ} \mathrm{C}$. The device was then washed with PBS and primed with DRG neuron media until plating. In the cell body compartment, 20,000 DRG neurons (dissected out from embryonic day 14 rats) were plated to attach at room temperature for $5 \mathrm{~min}$. DRG neurons were infected with lentivirus in the cell body compartment diluted in media containing $0.3 \mu \mathrm{M} \mathrm{AraC} ; 100 \mathrm{ng} / \mathrm{mL} \mathrm{NGF}+\mathrm{BDNF}$ and fresh media with $0.3 \mu \mathrm{M} \mathrm{AraC}$; $100 \mathrm{ng} / \mathrm{mL} \mathrm{NGF}+$ BDNF added to the distal axon compartment. At DIV1, virus was removed and replaced with fresh media to both compartments with the cell body neurotrophins reduced to $10 \mathrm{ng} / \mathrm{mL} \mathrm{NGF}+$ BDNF with AraC and distal axon compartment with $0.3 \mu \mathrm{M} \mathrm{AraC} ; 100 \mathrm{ng} / \mathrm{mL}$ NGF + BDNF. At DIV2, both cell body and distal axon compartment were kept in $0.3 \mu \mathrm{M} \mathrm{AraC} ; 5 \mathrm{ng} / \mathrm{mL} \mathrm{NGF}+$ BDNF until live cell 391 imaging at DIV5.

For immunofluorescence in microfluidic chambers, silicon-based 450 $\mu \mathrm{m}$ microgroove barrier (SND450; Xona) was prepared following manufacturer's instructions with modifications. Sterilized cover glass was coated with $0.2 \mathrm{mg} / \mathrm{mL}$ poly-D-lysine (Sigma) overnight, then washed with water and dried. Microfluidic chambers were cleaned by briefly soaking it in ethanol then dried; attached onto PDL-coated glass slide; and the chambers and the wells were filled with $10 \mathrm{ug} / \mathrm{mL}$ laminin and incubated for 3 hours at $37^{\circ} \mathrm{C}$. The chambers and wells were washed three times with neurobasal media and 30,000 DRG neurons were plated in the cell body compartment with $0.3 \mu \mathrm{M} \mathrm{AraC} ; 50 \mathrm{ng} / \mathrm{mL} \mathrm{NGF}+\mathrm{BDNF}$ and $0.3 \mu \mathrm{M} \mathrm{AraC}$; $100 \mathrm{ng} / \mathrm{mL} \mathrm{NGF}+$ BDNF in the distal axon compartment. At DIV1, both compartments were replaced with fresh media with $0.3 \mu \mathrm{M}$ AraC; $10 \mathrm{ng} / \mathrm{mL} \mathrm{NGF}+\mathrm{BDNF}$ in the cell body compartment and $0.3 \mu \mathrm{M} \mathrm{AraC}$; $100 \mathrm{ng} / \mathrm{mL}$ NGF + BDNF in the distal axon compartment and maintained until DIV5 for immunostaining.

403 See Fenstermacher et al. for more details on compartmented culture system (Fenstermacher, PazyraMurphy, \& Segal, 2015). 
Axonal degeneration assay: Compartmented chamber cultures were fixed at room temperature with $4 \%$ PFA diluted 1:2 in DRG neuron media for $10 \mathrm{~min}$, then an additional 20 minutes in undiluted 4\% PFA. DRGs were permeabilized with $0.1 \%$ Triton X-100 for 10 min; blocked in 3\% BSA in $0.1 \%$ Triton X-100 for 1 hour at room temperature; and incubated with rabbit anti-Tuj1 (1:400; Biolegend) overnight at $4^{\circ} \mathrm{C}$. Cultures were then incubated with secondary antibodies (1:1000; Invitrogen) for 1 hour at room temperature and stained briefly with DAPI. Images of distal axon tips were obtained using a 40X air objective, and axonal degeneration was quantified as a degeneration index, as previously described (Cosker et al., 2016; Sasaki, Vohra, Lund, \& Milbrandt, 2009). For peptide rescue experiment, the following peptides synthesized as previously described (Barclay et al., 2015; Pease-Raissi et al., 2017) were used:

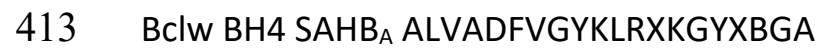

414 BCl2 BH4 SAHB AIVBKYIHYKLSXRGYXWDA (differential placement of all-hydrocarbon staples $(\mathrm{X})$ along the $\mathrm{BH} 4$ sequences of $\mathrm{Bclw}$ and $\mathrm{Bcl} 2$; and $\mathrm{B}$ represents norleucine, replacing the native cysteine and methionine on $B c l w$ and $B c / 2$, respectively)

417 The peptides were introduced into axons using $2 \mu \mathrm{L}$ Chariot protein transfection system (Active Motif) 418 only in the distal axon compartment of compartmented Campenot chambers immediately after R280H 419 virus removal at DIV2. The culture was kept with the peptides until DIV8 for axon degeneration assay.

420 Immunofluorescence: DRG neurons grown in silicon-based microfluidic chambers were fixed with 4\% PFA in PBS for 20 minutes at room temperature then washed three times with PBS. The device was then carefully removed and then cultures were immediately permeabilized with $0.1 \%$ triton in PBS for 10 minutes at room temperature and blocked for 1 hour at room temperature with 3\% BSA in PBS. KIF5 primary antibodies (1:100; abcam) and mouse TUJ1 (1:400; Biolegend) diluted in 3\% BSA in PBS were incubated at $4^{\circ} \mathrm{C}$ overnight. The slides were washed three times with PBS and secondary antibodies diluted in 3\% BSA in PBS (1:1000; Invitrogen) were incubated for 1 hour at room temperature. Finally, the slides were washed two times with PBS and incubated with DAPI (1:1000) in PBS; washed briefly and mounted. Images were acquired with Nikon C2 Si laser-scanning confocal microscope with 60x oil objective using NIS-Elements imaging software.

Whole mount immunostaining: Whole DRG with peripheral nerves were dissected from P1 mice of either sex and fixed with $4 \%$ PFA at $4^{\circ} \mathrm{C}$ overnight. DRGs were washed in PBS, permeabilized in $0.5 \%$ Triton X100 for 1 hour and blocked in 5\% BSA and 0.5\% Triton X-100 for 4 hours. DRGs were incubated for 48 hours in primary KIF5 (1:50, Abcam) and TUJ1 (1:300, Biolegend) antibodies at $4^{\circ} \mathrm{C}$ and washed overnight in PBS. DRGs were then incubated in secondary antibody (1:500; Life Technologies) at room temperature for 3.5 hours. Images were acquired with Nikon C2 Si laser-scanning confocal microscope with 40x oil 436 objective.

437 Live cell imaging: DRGs neurons infected with lentivirus expressing Halo-SFPQ were grown in XonaChip 438 microfluidic chambers and were labeled with tetramethyl Rhodamine (TMR) Halo Tag ligand (Promega) according to the manufacturer's instructions with modifications. TMR stock was diluted in DRG culture media at 1:200 and used at a final labeling concentration of $2.5 \mu \mathrm{M}$ added to both cell body and axon compartment. DRGs were incubated for 15 mins at $37^{\circ} \mathrm{C}$ and washed 3 times with complete culture media made with neurobasal without phenol red and incubated for 30 mins at $37^{\circ} \mathrm{C}$ to wash unbound ligand. DRG culture media was replaced with low fluorescence imaging media (HibernateE; Brain Bits) supplemented with 2\% B27 and 1\% GlutaMAX. DRG neurons were imaged live in an environmental chamber at $37^{\circ} \mathrm{C}$ and $7.5 \% \mathrm{CO}_{2}$ using a $60 \mathrm{x}$ oil $1.4 \mathrm{NA}$ objective with a Perfect Focus System one frame every $1.5 \mathrm{sec}$ for 3 mins. Images were analyzed using Kymolyzer macro for ImageJ developed by the 
laboratory of Dr. Thomas Schwarz (Shlevkov et al., 2019). To determine the SFPQ granule diameter, measurement was taken from the two edges of the granule running parallel to the direction of the axon.

Western blot: HEK 293T cells or DRG sensory neurons were collected and prepped with lysis buffer (1\% NP-40; $50 \mathrm{mM}$ Tris-HCl, pH 7.4; $150 \mathrm{mM} \mathrm{NaCl}$; $2 \mathrm{mM}$ EDTA; protease inhibitor (Sigma); and phosphatase inhibitor (Life Technologies)). Cell lysates were placed on ice for 20 minutes and centrifuged at 13,000 rpm for 20 minutes to collect the supernatant. The lysates were separated by $4-12 \%$ Bis-Tris NuPAGE gel (Thermo Fisher) and blotted with the following primary antibodies: mouse anti-SFPQ (1:1000; Sigma), rabbit anti-KIF5A (1:2000; Abcam), rabbit anti-KIF5B (1:2000; Abcam), rabbit anti-KIF5C (1:2000; Abcam), rabbit anti-KLC1 (1:500; Santa Cruz), rabbit anti-KLC2 (1:1000; Proteintech), rabbit anti-pan actin (1:1000; Cell Signaling), mouse anti-Myc (1:500; Santa Cruz), mouse anti-FLAG (1:1000; Sigma), mouse anti-HA (1:10,000; Thermo Fisher), rabbit anti-GFP (1:2000; Sigma). HRP-conjugated secondary antibodies (1:10,000; BioRad); ECL detection system (VWR) and SuperSignal West Dura (Thermo Fisher) were used for chemiluminescent detection.

Transfection and immunoprecipitation: HEK $293 \mathrm{~T}$ cells were cultured in a $10 \mathrm{~cm}$ plate with DMEM, 10\% FBS (Thomas Scientific) and $1 \%$ Penicillin/streptomycin at $37^{\circ} \mathrm{C}$ and $5 \% \mathrm{CO}_{2}$. For transfection, cells were plated in a $6 \mathrm{~cm}$ dish and 24 hours later plasmids were transfected with Lipofectamine 2000 (Invitrogen) based on manufacture's protocol and then incubated for 24 hours before immunoprecipitation experiments.

For immunoprecipitation, HEK 293T cell or DRG neurons were collected and lysed as described for western blots, and $500 \mu \mathrm{g}$ of protein lysate was precleared with $3 \mu \mathrm{L}$ of Dynabeads protein $\mathrm{G}$ (Thermo Fisher) for 1 (HEK 293T lysates) or 2 hours (DRG neuron lysates) nutated at $4^{\circ} \mathrm{C}$. For RNase experiments, the lysate was treated with RNase A/T1 (Fisher Scientific) for 1 hour at room temperature and immediately immunoprecipitated. Following the manufacturer's instructions, protein lysate was immunoprecipitated for 2 hours at $4^{\circ} \mathrm{C}$ with the following antibodies: rabbit anti-myc ( $2.5 \mu \mathrm{g}$, Millipore Sigma), mouse anti-HA $(2.5 \mu \mathrm{g}$, Thermo Fisher), mouse anti-SFPQ $(20 \mu \mathrm{g}$, Abcam), control normal mouse (Millipore Sigma) or normal rabbit IgG (Cell Signaling),. The input (0.5\% for HEK 293T and 3\% for DRG) and the elute was 473 analyzed by western blot.

Lentivirus production and infection: HEK 293T cells grown on $10 \mathrm{~cm}$ dish were transfected using FuGENE 6 (Promega) with the transfer vector pxPAX2 (Addgene \#12260) and pMD2.G (Addgene plasmid \#12259), gifts from Didier Trono, at a ratio of 4:3:1. The transfection reagent was replaced with fresh media after 24 hours. Virus-containing media were collected 48- and 72-hours post transfection; pooled; centrifuged at $1200 \mathrm{rpm}$ for 5 minutes; and filtered through a $0.45 \mu \mathrm{m}$ PES filter. Finally, the virus was concentrated using Amicon ultra-15 centrifugal filter units (Millipore Sigma) by centrifuging at $3000 \mathrm{rpm}$ and stored at $-80^{\circ} \mathrm{C}$ until use. For infection of DRG sensory neurons, virus was added at DIV1 for 24 hours, except for XonaChip where virus was added immediately after plating. For experiments involving puromycin (Sigma) selection (Figure 6D, shSFPQ; Figure 6F, KIF5A WT and R280H constructs; Figure 2-figure supplement 1C and 1D, shKIF5A and shKIF5C; Figure 6-figure supplement 1, shSFPQ), the neurons were allowed to recover for 1 day after virus removal and $0.4 \mu \mathrm{g} / \mathrm{mL}$ puromycin was then added at DIV3 and replaced with fresh media at DIV5.

Protein expression and purification: A construct of human KNS2 covering residues 205-501 in the pET28MHL vector, a gift from Cheryl Arrowsmith (Addgene plasmid \#26096) was expressed in E. coli BL21 (DE3) in TB medium in the presence of $50 \mu \mathrm{g} / \mathrm{mL}$ of kanamycin. Cells were grown at $37^{\circ} \mathrm{C}$ to an OD of 0.6 , induced overnight at $17^{\circ} \mathrm{C}$ with $400 \mu \mathrm{M}$ isopropyl-1-thio-D-galactopyranoside, collected by centrifugation, 
and stored at $-80^{\circ} \mathrm{C}$. Cell pellets were microfluidized at 15,000 psi in buffer $\mathrm{A} 1$ ( $25 \mathrm{mM}$ HEPES (7.5), 500mM $\mathrm{NaCl}, 5 \%$ glycerol, $30 \mathrm{mM}$ Imidazole, $5 \mathrm{uM} \mathrm{ZnAc}$, and $7 \mathrm{mM} \mathrm{BME}$ ) and the resulting lysate was centrifuged at 13,000 rpm for $40 \mathrm{~min}$. Ni-NTA beads (Qiagen) were mixed with lysate supernatant for $45 \mathrm{~min}$, washed with buffer A1, and eluted with buffer Bi ( $25 \mathrm{mM} \mathrm{HEPES} \mathrm{(7.5),} 500 \mathrm{mM} \mathrm{NaCl}, 5 \%$ glycerol, $400 \mathrm{mM}$ Imidazole, $5 \mathrm{uM} Z \mathrm{ZnAc}$, and $7 \mathrm{mM}$ BME). The sample was gel-filtered through a Superdex-200 16/60 column in buffer A3 (20mM HEPES (7.5), $200 \mathrm{mM} \mathrm{NaCl}, 5 \%$ glycerol, $1 \mathrm{mM}$ DTT, and $0.5 \mathrm{mM}$ TCEP). Fractions were pooled, but protein began precipitating when concentrated. To combat apparent precipitation, excess $\mathrm{NaCl}$ solution was added to a final buffer composition of $18 \mathrm{mM}$ HEPES-7.5, $680 \mathrm{mM} \mathrm{NaCl}, 4.5 \%$ glycerol, $0.9 \mathrm{mM}$ $\mathrm{DTT}$, and $0.45 \mathrm{mM}$ TCEP. Adjusted sample was then concentrated and stored at $-80^{\circ} \mathrm{C}$.

Isothermal Titration Calorimetry (ITC): All calorimetric experiments were carried out in 20mM HEPES, pH $7.5,150 \mathrm{mM} \mathrm{NaCl}$, and $0.5 \mathrm{mM} \mathrm{TCEP}$, with $2 \%$ DMSO at $25^{\circ} \mathrm{C}$ using an Affinity ITC from TA Instruments (New Castle, DE) equipped with auto sampler. Briefly, $350 \mu \mathrm{L}$ of buffer or protein at $20 \mu \mathrm{M}$ was placed into the calorimetric cell, and $250 \mu \mathrm{L}$ of various SFPQ peptides (KE BioChem) at $200 \mu \mathrm{M}$ were loaded into titration syringe. $4 \mu \mathrm{L}$ syringe solution was injected into the calorimetric cell 30 times with a 200 second interval between injections. Thermodynamic parameters ( $\mathrm{Kd}$, stoichiometry, and enthalpy) were calculated according to the single site model provided in NanoAnalyze software (TA instruments).

Mass Spectrometry: Antibody-conjugated protein G beads from KLC1 and SFPQ immunoprecipitates were suspended in $100 \mu \mathrm{L}$ of ammonium bicarbonate, reduced with $10 \mathrm{mM}$ dithio-treitol (DTT) for 30 minutes at $56^{\circ} \mathrm{C}$ and alkylated with $20 \mathrm{mM}$ iodoacetamide for 20 minutes at $22^{\circ} \mathrm{C}$ in the dark. Excess iodoacetamide was quenched by adding $10 \mathrm{mM}$ dithio-treitol (DTT) before diluting the samples to $250 \mu \mathrm{L}$ with $100 \mathrm{mM}$ ammonium bicarbonate. Immunoprecipitated proteins were digested overnight at $37^{\circ} \mathrm{C}$ with $4 \mu \mathrm{g}$ of trypsin. Tryptic peptides were desalted using $500 \mu \mathrm{g}$ of a 1:1 mixture of hydrophobic and hydrophilic Sera-

Peptides were loaded onto a precolumn (100 $\mu \mathrm{m} \times 4 \mathrm{~cm}$ POROS 10R2, Applied Biosystems) and eluted with an HPLC gradient (NanoAcquity UPLC system, Waters; $1 \%-40 \% \mathrm{~B}$ in 90 min; $\mathrm{A}=0.2 \mathrm{M}$ acetic acid in water, $B=0.2 \mathrm{M}$ acetic acid in acetonitrile). Peptides were resolved on a self-packed analytical column ( 30 $\mu \mathrm{m} \times 50 \mathrm{~cm}$ Monitor C18, Column Engineering) and introduced in the mass spectrometer (QExactive HF mass spectrometer, ThermoFisher Scientific) equipped with a Digital PicoView electrospray source platform (New Objective)(Ficarro et al., 2009).

The mass spectrometer was programmed to perform a combination of targeted (Parallel Reaction Monitoring, PRM) and data dependent MS/MS scans. To select precursors for the PRM experiments, we first analyzed a small aliquot of digested KLC1 immunoprecipitate and selected the most intense precursor for peptides mapping uniquely to genes (Askenazi, Marto, \& Linial, 2010) encoding each protein of interest. In data-dependent mode, the top 5 most abundant ions in each MS scan were subjected to collision induced dissociation (HCD, 27\% normalized collision energy) MS/MS (isolation width $=1.5 \mathrm{Da}$, intensity threshold $=1 \mathrm{E} 5$, max injection time: $50 \mathrm{~ms}$ ). Dynamic exclusion was enabled with an exclusion duration of 30 seconds. PRM scans were scheduled across an 8 minute-period for each of 45 precursors selected as described above (isolation width $=1.6 \mathrm{Da}$, max injection time: $119 \mathrm{~ms}$ ). ESI voltage was set to $3.8 \mathrm{kV}$.

MS spectra were recalibrated using the background ion $(\mathrm{Si}(\mathrm{CH} 3) 20) 6$ at $\mathrm{m} / \mathrm{z} 445.12+/-0.03$ and converted into a Mascot generic file format (.mgf) using multiplierz scripts (Alexander, Ficarro, Adelmant, \& Marto, 2017; Askenazi, Parikh, \& Marto, 2009; Parikh et al., 2009). Spectra were searched using Mascot (version 2.6) against three appended databases consisting of: i) rat protein sequences (downloaded from UniProt 538 on 04/09/2018); ii) common lab contaminants and iii) a decoy database generated by reversing the 
sequences from these two databases. Precursor tolerance was set to $20 \mathrm{ppm}$ and product ion tolerance to $25 \mathrm{mmu}$. Search parameters included trypsin specificity, up to 2 missed cleavages, fixed carbamidomethylation $(C,+57 \mathrm{Da})$ and variable oxidation $(\mathrm{M},+16 \mathrm{Da})$. Spectra matching to peptides from the reverse database were used to calculate a global false discovery rate and were discarded. Data were further processed to remove peptide spectral matches (PSMs) to the forward database with an FDR greater than 1.0\%. Protein abundance for KLC1 and KLC2 (Fig. 1G) or KIF5A, KIF5B, KIF5C (Fig. 1H), in the KLC1 immunoprecipitate was calculated by summing the extracted ion chromatogram peak area of the 3 most abundant (Silva, Gorenstein, Li, Vissers, \& Geromanos, 2006) gene-unique peptide sequences and averaged across 2 technical replicates. Due to the low absolute abundance of KLC and KIF proteins in the SFPQ immunoprecipitates, we used an MS2-level quantitation approach whereby the extracted ion chromatogram intensity (calculated as the area under the curve) of a set of precursor/fragment ion pairs (manually selected from the targeted MS/MS experiments) in the SFPQ LC-MS/MS analyses were normalized to their intensity in the KLC1 immunoprecipitate, taking into account the average abundance of each KLC (Fig. 1G) or KIF5 (Fig. 1H) protein measured by the top3 quantitation method described above.

\section{Quantification and Statistical Analysis}

555 Data are expressed as mean \pm s.e.m. To assess statistical significance, data were analyzed by unpaired was placed at $p<0.05$. Statistical analysis was done using Microsoft Excel and GraphPad Prism. 


\section{References}

Alexander, W. M., Ficarro, S. B., Adelmant, G., \& Marto, J. A. (2017). multiplierz v2.0: A Pythonbased ecosystem for shared access and analysis of native mass spectrometry data. Proteomics, 17(15-16). doi:10.1002/pmic.201700091

Askenazi, M., Marto, J. A., \& Linial, M. (2010). The complete peptide dictionary--a metaproteomics resource. Proteomics, 10(23), 4306-4310. doi:10.1002/pmic.201000270

Askenazi, M., Parikh, J. R., \& Marto, J. A. (2009). mzAPI: a new strategy for efficiently sharing mass spectrometry data. Nat Methods, 6(4), 240-241. doi:10.1038/nmeth0409-240

Barclay, L. A., Wales, T. E., Garner, T. P., Wachter, F., Lee, S., Guerra, R. M., ... Walensky, L. D. (2015). Inhibition of Pro-apoptotic BAX by a noncanonical interaction mechanism. Mol Cell, 57(5), 873-886. doi:10.1016/j.molcel.2015.01.014

Cosker, K. E., Fenstermacher, S. J., Pazyra-Murphy, M. F., Elliott, H. L., \& Segal, R. A. (2016). The RNA-binding protein SFPQ orchestrates an RNA regulon to promote axon viability. Nat Neurosci, 19(5), 690-696. doi:10.1038/nn.4280

Das, S., Singer, R. H., \& Yoon, Y. J. (2019). The travels of mRNAs in neurons: do they know where they are going? Curr Opin Neurobiol, 57, 110-116. doi:10.1016/j.conb.2019.01.016

Dutta, M., Diehl, M. R., Onuchic, J. N., \& Jana, B. (2018). Structural consequences of hereditary spastic paraplegia disease-related mutations in kinesin. Proc Natl Acad Sci U S A, 115(46), E10822-E10829. doi:10.1073/pnas.1810622115

Ebbing, B., Mann, K., Starosta, A., Jaud, J., Schols, L., Schule, R., \& Woehlke, G. (2008). Effect of spastic paraplegia mutations in KIF5A kinesin on transport activity. Hum Mol Genet, 17(9), 1245-1252. doi:10.1093/hmg/ddn014

Fenstermacher, S. J., Pazyra-Murphy, M. F., \& Segal, R. A. (2015). Campenot Cultures and Microfluidics Provide Complementary Platforms for Spatial Study of Dorsal Root Ganglia Neurons. In Microfluidic and Compartmentalized Platforms for Neurobiological Research (pp. 105-124).

Ficarro, S. B., Zhang, Y., Lu, Y., Moghimi, A. R., Askenazi, M., Hyatt, E., . . Marto, J. A. (2009). Improved electrospray ionization efficiency compensates for diminished chromatographic resolution and enables proteomics analysis of tyrosine signaling in embryonic stem cells. Anal Chem, 81(9), 3440-3447. doi:10.1021/ac802720e

Fuger, P., Sreekumar, V., Schule, R., Kern, J. V., Stanchev, D. T., Schneider, C. D., .. Rasse, T. M. (2012). Spastic paraplegia mutation N256S in the neuronal microtubule motor KIF5A disrupts axonal transport in a Drosophila HSP model. PLoS Genet, 8(11), e1003066. doi:10.1371/journal.pgen.1003066

Gopal, P. P., Nirschl, J. J., Klinman, E., \& Holzbaur, E. L. (2017). Amyotrophic lateral sclerosislinked mutations increase the viscosity of liquid-like TDP-43 RNP granules in neurons. Proc Natl Acad Sci $U S S$ A, 114(12), E2466-E2475. doi:10.1073/pnas.1614462114

Hayer, A., Shao, L., Chung, M., Joubert, L. M., Yang, H. W., Tsai, F. C., . . Meyer, T. (2016). Engulfed cadherin fingers are polarized junctional structures between collectively migrating endothelial cells. Nat Cell Biol, 18(12), 1311-1323. doi:10.1038/ncb3438

Hewage, T. W., Caria, S., \& Lee, M. (2019). A new crystal structure and small-angle X-ray scattering analysis of the homodimer of human SFPQ. Acta Crystallogr F Struct Biol Commun, 75(Pt 6), 439-449. doi:10.1107/S2053230X19006599 
604

605

606

607

608

609

610

611

612

613

614

615

616

617

618

619

620

621

622

623

624

625

626

627

628

629

630

631

632

633

634

635

636

637

638

639

640

641

642

643

644

645

646

647
Jennings, S., Chenevert, M., Liu, L., Mottamal, M., Wojcik, E. J., \& Huckaba, T. M. (2017). Characterization of kinesin switch I mutations that cause hereditary spastic paraplegia. PLoS One, 12(7), e0180353. doi:10.1371/journal.pone.0180353

Kanai, Y., Dohmae, N., \& Hirokawa, N. (2004). Kinesin transports RNA: isolation and characterization of an RNA-transporting granule. Neuron, 43(4), 513-525. doi:10.1016/j.neuron.2004.07.022

Knowles, R. B., Sabry, J. H., Martone, M. E., Deerinck, T. J., Ellisman, M. H., Bassell, G. J., \& Kosik, K. S. (1996). Translocation of RNA granules in living neurons. J Neurosci, 16(24), 7812-7820. Retrieved from https://www.ncbi.nlm.nih.gov/pubmed/8987809

Liao, Y. C., Fernandopulle, M. S., Wang, G., Choi, H., Hao, L., Drerup, C. M., .. Ward, M. E. (2019). RNA Granules Hitchhike on Lysosomes for Long-Distance Transport, Using Annexin A11 as a Molecular Tether. Cell, 179(1), 147-164 e120. doi:10.1016/j.cell.2019.08.050

Lipka, J., Kapitein, L. C., Jaworski, J., \& Hoogenraad, C. C. (2016). Microtubule-binding protein doublecortin-like kinase 1 (DCLK1) guides kinesin-3-mediated cargo transport to dendrites. EMBO J, 35(3), 302-318. doi:10.15252/embj.201592929

Liu, Y. T., Laura, M., Hersheson, J., Horga, A., Jaunmuktane, Z., Brandner, S., . . Houlden, H. (2014). Extended phenotypic spectrum of KIF5A mutations: From spastic paraplegia to axonal neuropathy. Neurology, 83(7), 612-619. doi:10.1212/WNL.0000000000000691

Lois, C., Hong, E. J., Pease, S., Brown, E. J., \& Baltimore, D. (2002). Germline transmission and tissue-specific expression of transgenes delivered by lentiviral vectors. Science, 295(5556), 868-872. doi:10.1126/science.1067081

Luisier, R., Tyzack, G. E., Hall, C. E., Mitchell, J. S., Devine, H., Taha, D. M., ... Patani, R. (2018). Intron retention and nuclear loss of SFPQ are molecular hallmarks of ALS. Nat Commun, 9(1), 2010. doi:10.1038/s41467-018-04373-8

McCart, A. E., Mahony, D., \& Rothnagel, J. A. (2003). Alternatively spliced products of the human kinesin light chain 1 (KNS2) gene. Traffic, 4(8), 576-580. Retrieved from https://www.ncbi.nlm.nih.gov/pubmed/12839500

Millecamps, S., \& Julien, J. P. (2013). Axonal transport deficits and neurodegenerative diseases. Nat Rev Neurosci, 14(3), 161-176. doi:10.1038/nrn3380

Nam, D. E., Yoo, D. H., Choi, S. S., Choi, B. O., \& Chung, K. W. (2018). Wide phenotypic spectrum in axonal Charcot-Marie-Tooth neuropathy type 2 patients with KIF5A mutations. Genes Genomics, 40(1), 77-84. doi:10.1007/s13258-017-0612-x

Nguyen, T. Q., Aumont-Nicaise, M., Andreani, J., Velours, C., Chenon, M., Vilela, F., ... Menetrey, J. (2018). Characterization of the binding mode of JNK-interacting protein 1 (JIP1) to kinesin-light chain 1 (KLC1). J Biol Chem, 293(36), 13946-13960. doi:10.1074/jbc.RA118.003916

Nicolas, A., Kenna, K. P., Renton, A. E., Ticozzi, N., Faghri, F., Chia, R., . . Landers, J. E. (2018). Genome-wide Analyses Identify KIF5A as a Novel ALS Gene. Neuron, 97(6), 12681283 e1266. doi:10.1016/j.neuron.2018.02.027

Parikh, J. R., Askenazi, M., Ficarro, S. B., Cashorali, T., Webber, J. T., Blank, N. C., ... Marto, J. A. (2009). multiplierz: an extensible API based desktop environment for proteomics data analysis. BMC Bioinformatics, 10, 364. doi:10.1186/1471-2105-10-364 
Pease-Raissi, S. E., Pazyra-Murphy, M. F., Li, Y., Wachter, F., Fukuda, Y., Fenstermacher, S. J., . .. Segal, R. A. (2017). Paclitaxel Reduces Axonal Bclw to Initiate IP3R1-Dependent Axon Degeneration. Neuron, 96(2), 373-386 e376. doi:10.1016/j.neuron.2017.09.034 Pernigo, S., Chegkazi, M. S., Yip, Y. Y., Treacy, C., Glorani, G., Hansen, K., . . Steiner, R. A. (2018). Structural basis for isoform-specific kinesin-1 recognition of Y-acidic cargo adaptors. Elife, 7. doi:10.7554/eLife.38362

Sasaki, Y., Vohra, B. P., Lund, F. E., \& Milbrandt, J. (2009). Nicotinamide mononucleotide adenylyl transferase-mediated axonal protection requires enzymatic activity but not increased levels of neuronal nicotinamide adenine dinucleotide. J Neurosci, 29(17), 5525-5535. doi:10.1523/JNEUROSCI.5469-08.2009

Shlevkov, E., Basu, H., Bray, M. A., Sun, Z., Wei, W., Apaydin, K., . . Schwarz, T. L. (2019). A High-Content Screen Identifies TPP1 and Aurora B as Regulators of Axonal Mitochondrial Transport. Cell Rep, 28(12), 3224-3237 e3225. doi:10.1016/j.celrep.2019.08.035

Silva, J. C., Gorenstein, M. V., Li, G. Z., Vissers, J. P., \& Geromanos, S. J. (2006). Absolute quantification of proteins by LCMSE: a virtue of parallel MS acquisition. Mol Cell Proteomics, 5(1), 144-156. doi:10.1074/mcp.M500230-MCP200

Sleigh, J. N., Rossor, A. M., Fellows, A. D., Tosolini, A. P., \& Schiavo, G. (2019). Axonal transport and neurological disease. Nat Rev Neurol. doi:10.1038/s41582-019-0257-2

Thomas-Jinu, S., Gordon, P. M., Fielding, T., Taylor, R., Smith, B. N., Snowden, V., ... Houart, C. (2017). Non-nuclear Pool of Splicing Factor SFPQ Regulates Axonal Transcripts Required for Normal Motor Development. Neuron, 94(2), 322-336 e325. doi:10.1016/j.neuron.2017.03.026

Yamazaki, T., Souquere, S., Chujo, T., Kobelke, S., Chong, Y. S., Fox, A. H., . . Hirose, T. (2018). Functional Domains of NEAT1 Architectural lncRNA Induce Paraspeckle Assembly through Phase Separation. Mol Cell, 70(6), 1038-1053 e1037. doi:10.1016/j.molcel.2018.05.019

Zhu, H., Lee, H. Y., Tong, Y., Hong, B. S., Kim, K. P., Shen, Y., . . Park, H. W. (2012). Crystal structures of the tetratricopeptide repeat domains of kinesin light chains: insight into cargo recognition mechanisms. PLoS One, 7(3), e33943. doi:10.1371/journal.pone.0033943 
Figures with Legends
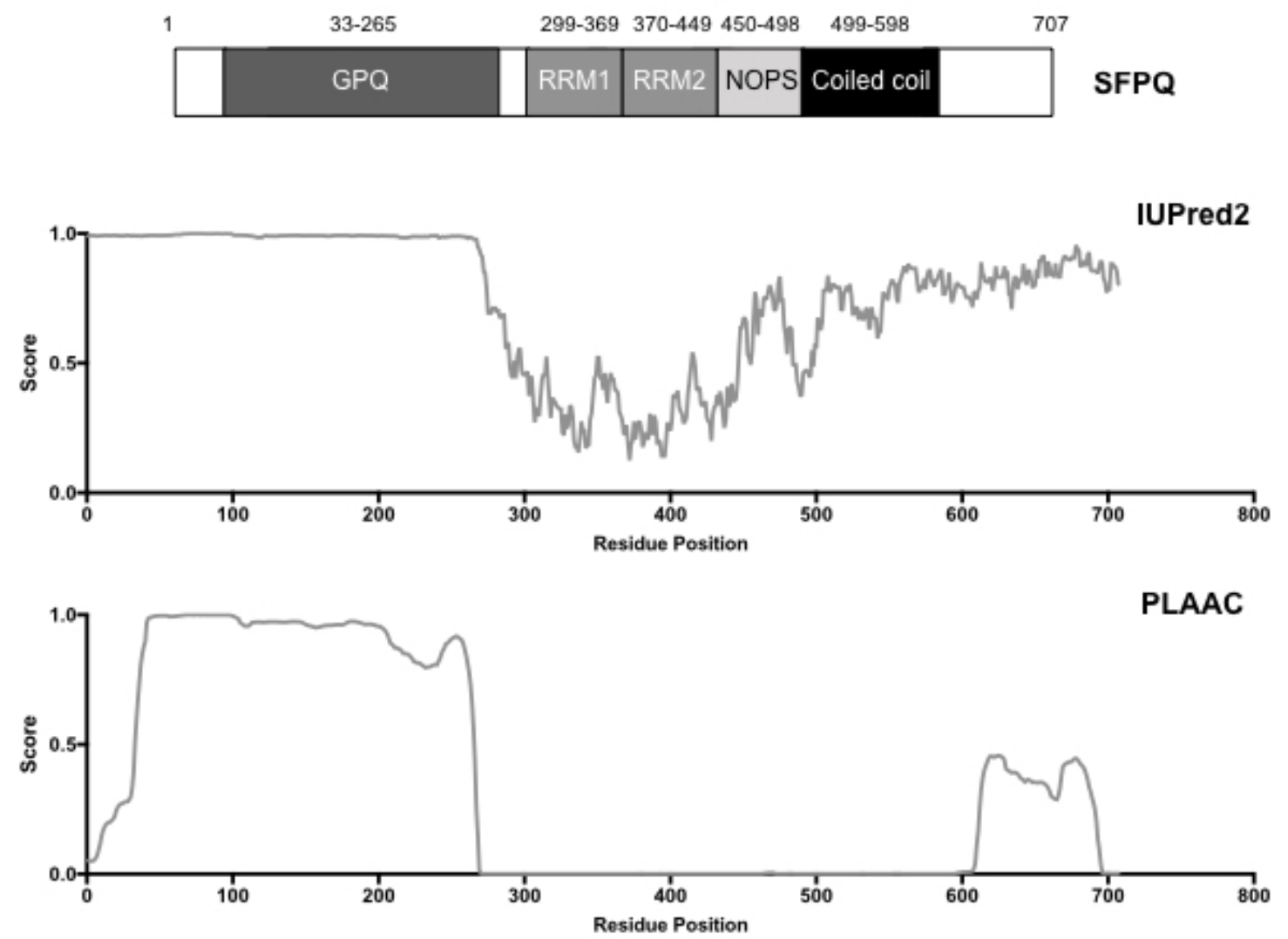

682

683

Introduction-figure supplement 1. Bioinformatic analysis of SFPQ protein sequence.

684

Schematic of protein domains of SFPQ and the probability score of SFPQ sequence for being disordered

685

686 and prion-like as predicted by IUPred2 (top) and PLAAC (bottom), respectively.

687 


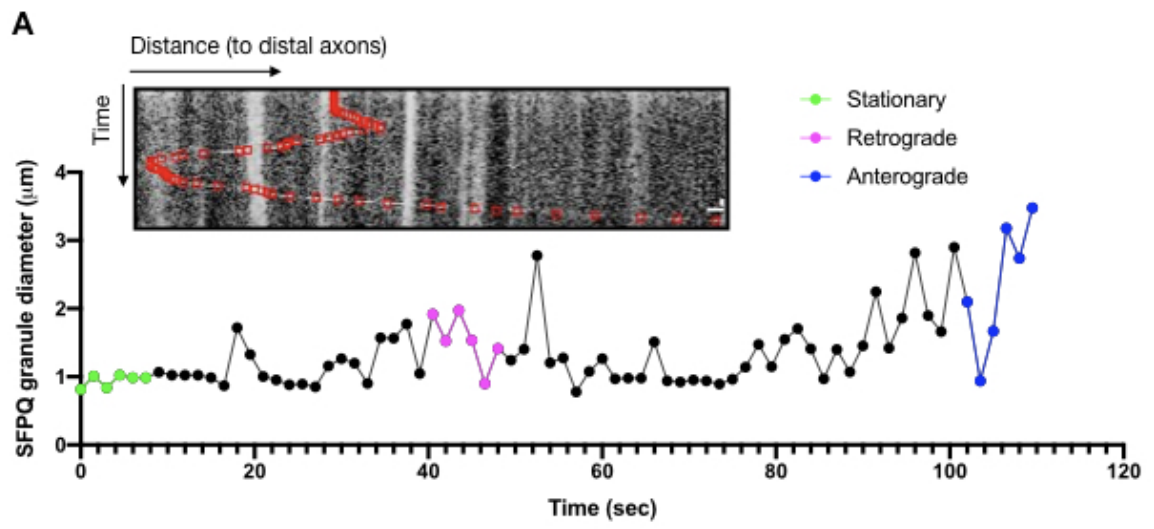

B

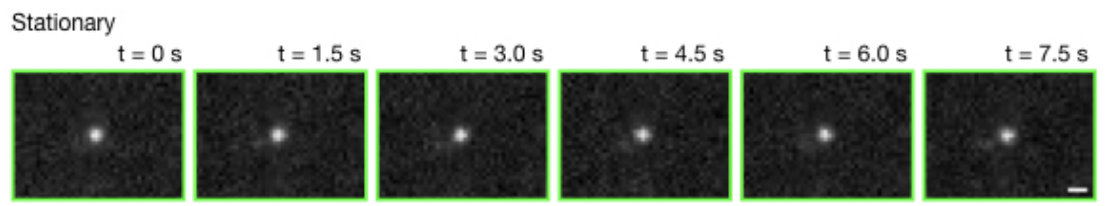

Retrograde
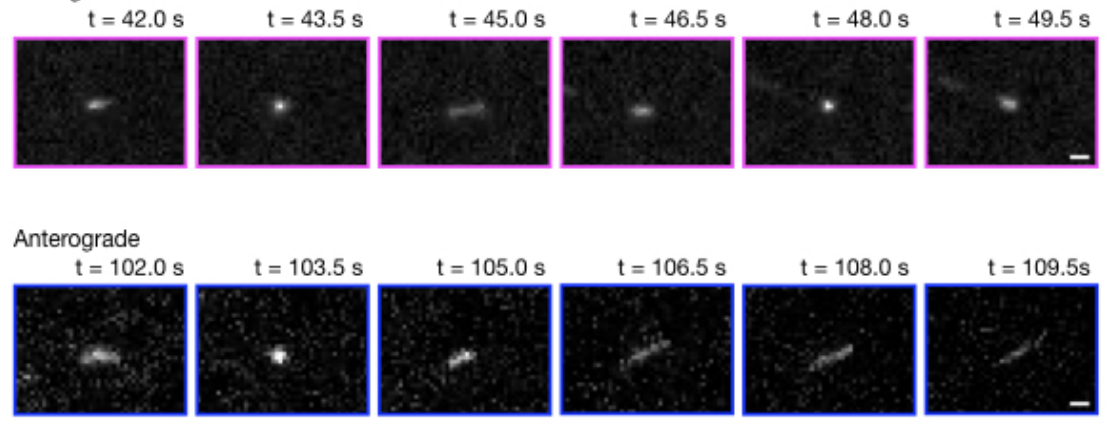

C

D
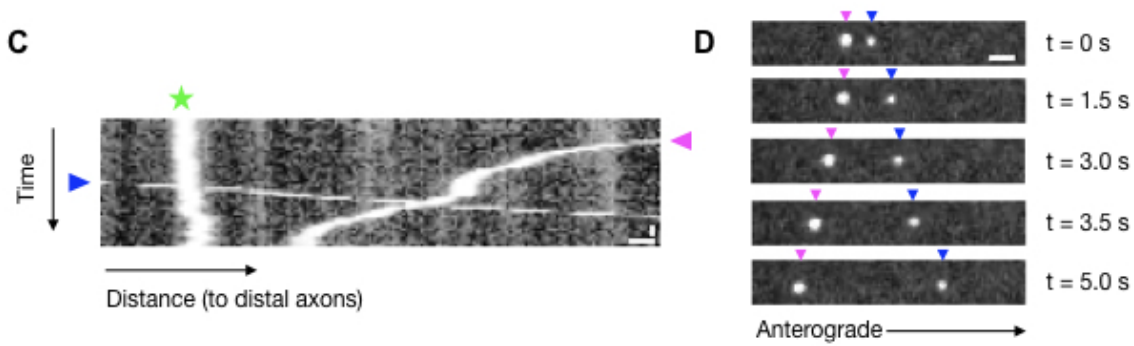

Figure 1. SFPQ granule, a non-membrane enclosed organelle, undergoes fast axonal transport.

(A) Kymograph of a Halo-SFPQ granule transitioning through stationary, retrograde and anterograde transport and its diameter plotted over time. Scale bars: $2 \mu \mathrm{m}$ and $6 \mathrm{sec}$. (B) Time-lapse images of the corresponding Halo-SFPQ granule from (A) in stationary (green), retrograde (magenta) and anterograde (blue) phase. Scale bars: $1 \mu \mathrm{m}$. (C) Kymograph depicting representative Halo-SFPQ in stationary (green star), anterograde (blue arrowhead) and retrograde (magenta arrowhead) phase. Scale bars: $2 \mu \mathrm{m}$ and 6 sec. (D) Representative time-lapse images of Halo-SFPQ in axons of DRG sensory neurons moving in anterograde (blue arrowhead) or retrograde (magenta arrowhead) direction. Scale bar: $2 \mu \mathrm{m}$.

Figure 1-figure supplement 1. Transport kinetics of SFPQ granules in axons of DRG sensory neurons. 
A

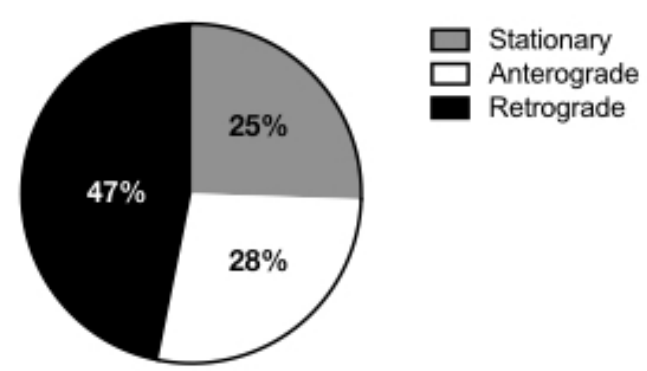

Fraction of SFPQ granules (\%)
B

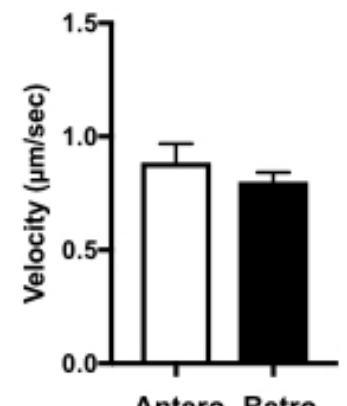

Antero. Retro.
C

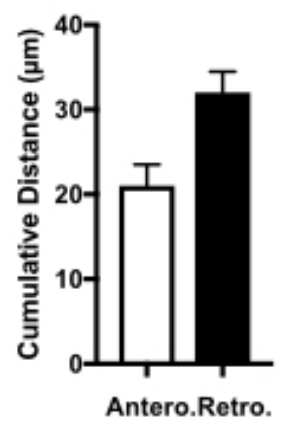

D
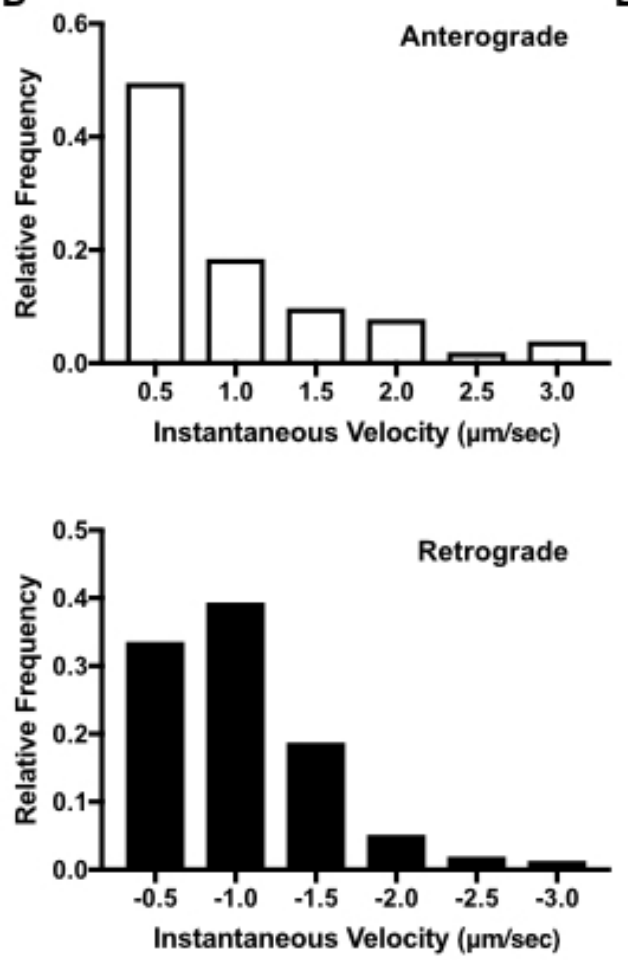

E
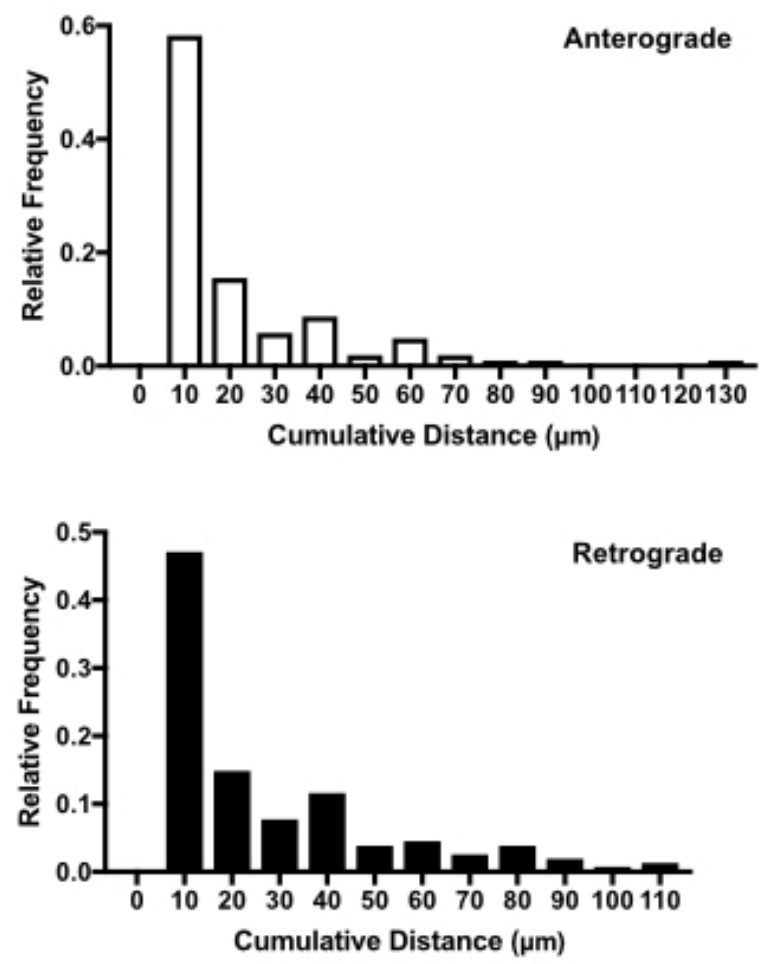

Figure 1-figure supplement 1. Transport kinetics of SFPQ granules in axons of DRG sensory neurons.

701
(A) Fraction of SFPQ granules spent in stationary, anterograde or retrograde phase. Data analyzed from 217 particles, from 29 axons, across 2 independent experiments. (B) Average velocity and (C) average cumulative distance of Halo-tagged SFPQ granules in axons and its frequency distribution in (D) and (E), respectively. 
A

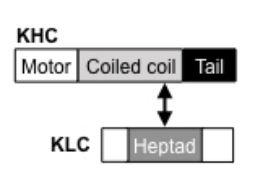

D

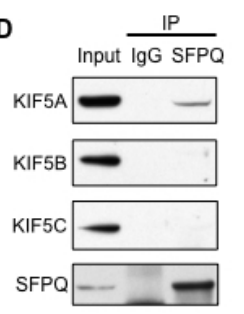

G

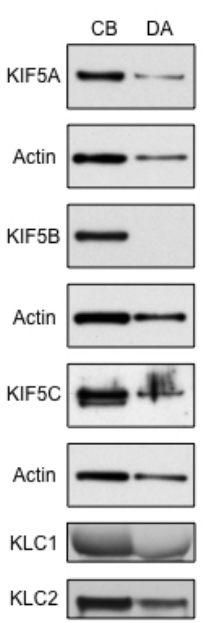

B

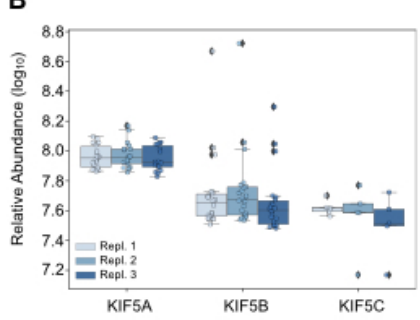

C

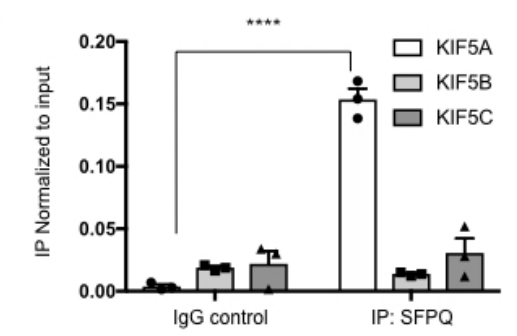

F

H
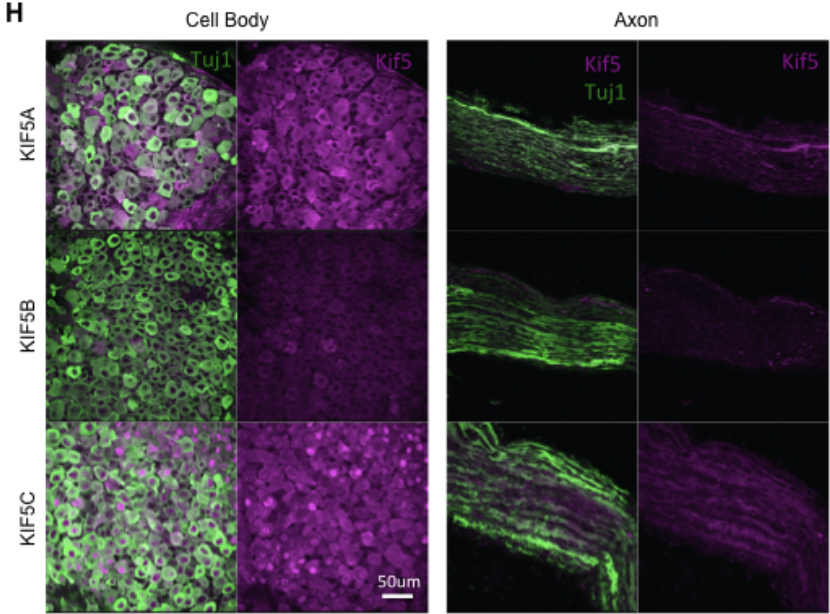

Figure 2. SFPQ preferentially binds to KIF5A/KLC1 motor complex.

(A) Schematic of domains of kinesin heavy chain (KHC) and kinesin light chain (KLC) and the interacting region between the heptad repeat of KLC and the coiled coil of KHC. ( $B$ and $C$ ) Box and whisker plot showing the relative abundance of KIF5A, KIF5B and KIF5C (B); and KLC1 and KLC2 (C) peptides derived from parallel reaction monitoring mass spectrometry. Data were acquired across three independent SFPQ immunoprecipitations (IPS). (D) IP of endogenous SFPQ from DRG sensory neuron protein lysate and blotted against endogenous KIF5A, KIF5B and KIF5C; IgG serves as control IP. (E) Quantification of pull down in (D) relative to input; $* * * * p<0.0001$ by one way ANOVA; $\mathrm{n}=3$ independent IPs; data represent mean \pm s.e.m. (F) IP of endogenous SFPQ from DRG sensory neuron protein lysate and blotted against endogenous KLC1 and KLC2; IgG serves as control IP. (G) Western blot of DRG neuron lysates of cell body (CB) and distal axons (DA) prepared from compartmented Campenot cultures probed against endogenous KIF5A, KIF5B, KIF5C, KLC1 and KLC2; actin serves as loading control. (H) Representative staining of endogenous KIF5A, KIF5B and KIF5C in DRGs and sciatic nerve of P1 mice; $n=4$ independent staining of tissues; scale bar $50 \mu \mathrm{m}$; Tuj1 (Green), KIF5 (Magenta).

Figure 2-figure supplement 1. Silver stain analysis of endogenous KLC1 and SFPQ IPs from DRGs and verification of antibodies for KIF5A, KIF5B and KIF5C.

Figure 2-figure supplement 2. KIF5 motors differentially localizes to cell body and distal axons. 
A

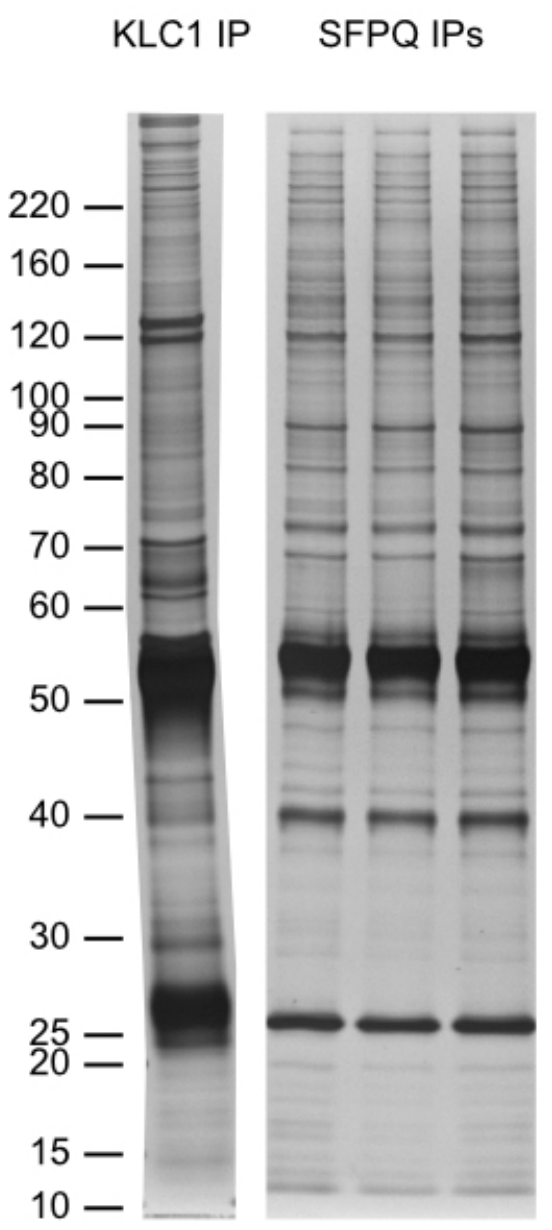

B

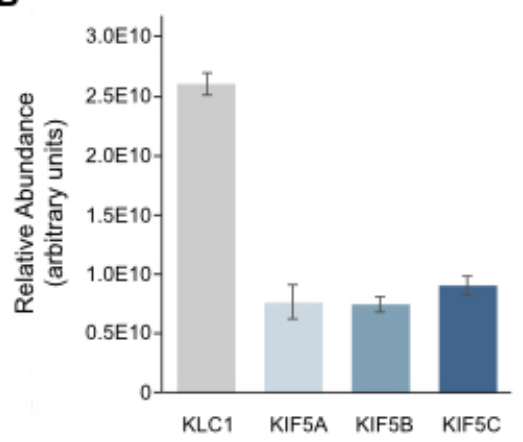

C

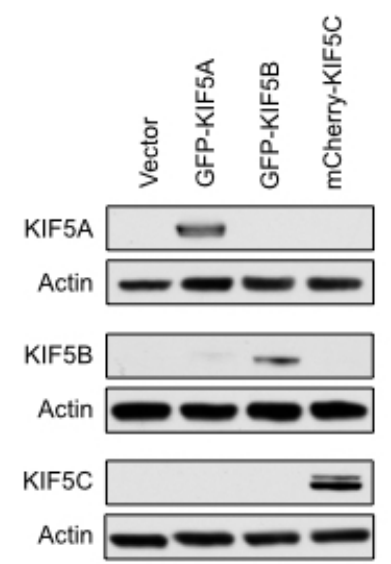

D

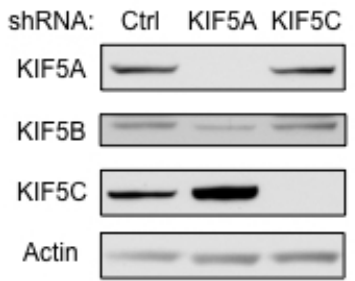

Figure 2-figure supplement 1. Silver stain analysis of endogenous KLC1 and SFPQ IPs from DRGs and verification of antibodies for KIF5A, KIF5B and KIF5C.

(A) Silver stain analysis of endogenous KLC1 and SFPQ proteins purified from DRG extracts. Each lane corresponds to $50 \%$ of the enriched material analyzed in Figure 2-figure supplement $1 B(K L C 1$ IP) and Figure 2B and 2C (three independent SFPQ IPs). (B) Relative abundance of KIF5A, KIF5B and KIF5C proteins (with KLC1 as a reference) in a KLC1 IP measured by label-free mass spectrometry. Data represent mean \pm s.e.m across two technical replicate analyses. (C) Western blot of HEK 293T lysates transfected with empty vector, GFP-KIF5A, GFP-KIF5B or mCherry-KIF5C and probed with the KIF5 antibodies. Actin serves as loading control. (D) DRG sensory neuron lysates infected with control (Ctrl) or with either shRNA against KIF5A or KIF5C and probed with the KIF5 antibodies. Actin serves as loading control. 

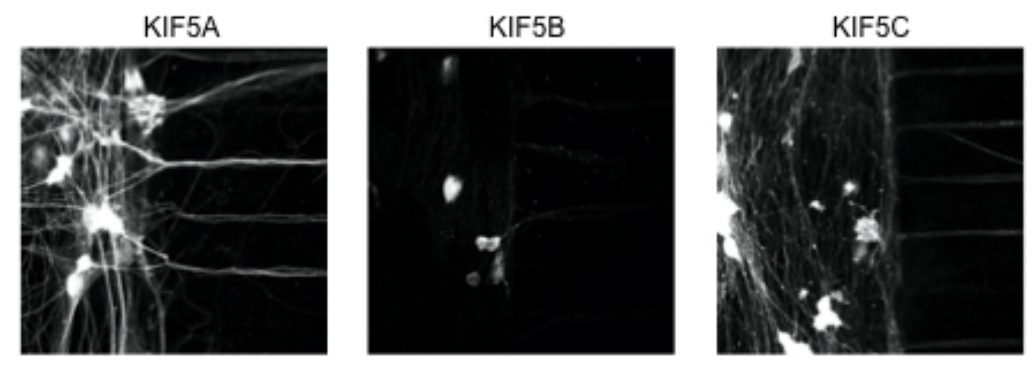

KIF5A TUJ1
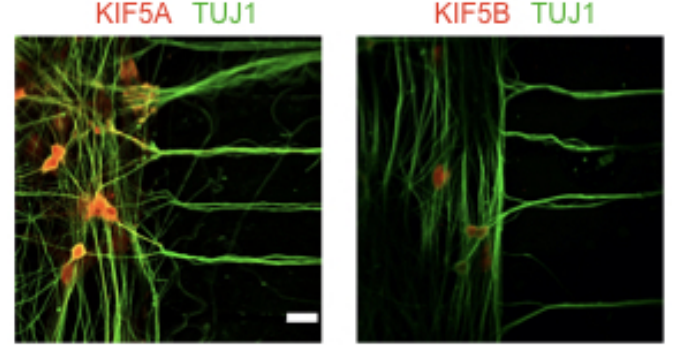

KIF5C TUJ1

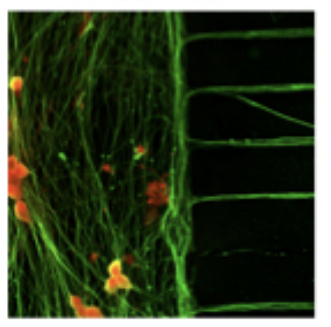

Figure 2-figure supplement 2. KIF5 motors differentially localize to cell body and distal axons. Representative staining of endogenous KIF5A, KIF5B and KIF5C in DRG sensory neurons grown in microfluidic chambers; Scale bar $20 \mu \mathrm{m}$; TUJ1 (green), KIF5 (Red). 
A

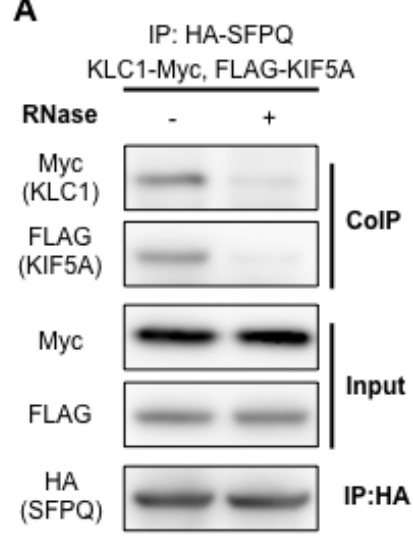

B

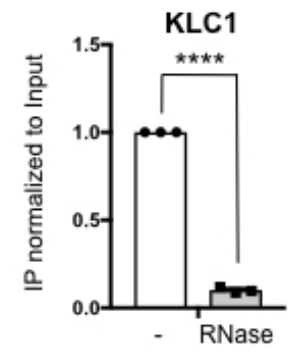

C

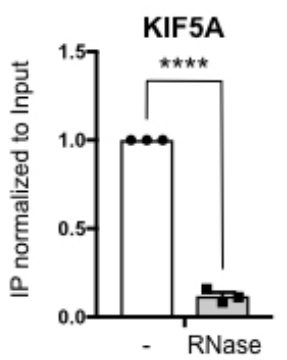

D

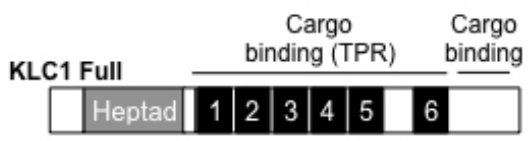

KLC1 $\Delta$ Tail (1-495)

\begin{tabular}{|l|l|l|l|l|l|l|l|l|l|l|l|l|l}
\hline Heptad & 1 & 2 & 3 & 4 & 5 & 6
\end{tabular}

E

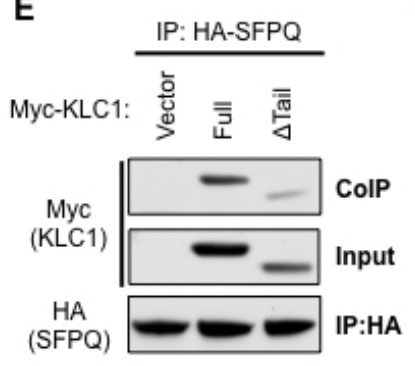

$\mathbf{F}$

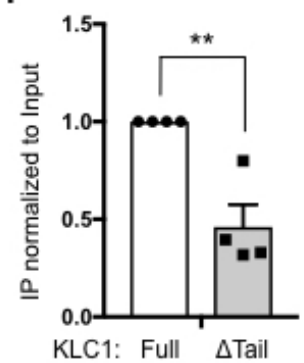

G

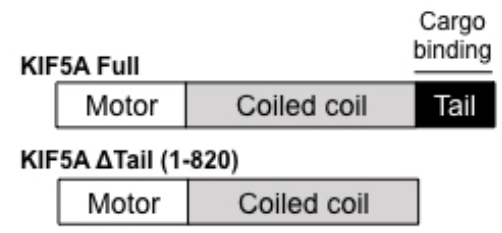

H

IP: HA-SFPQ

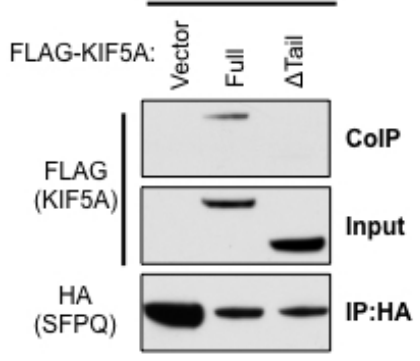

I

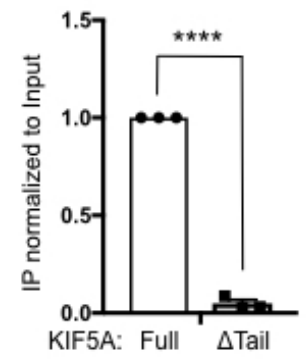

Figure 3. RNase prevents SFPQ-RNA binding to KIF5A/KLC1.

(A) HEK 293T cells transfected with HA-SFPQ, FLAG-KIF5A and KLC1-Myc and lysates were treated with or without RNase. HA was IPed and blotted for HA, Myc and FLAG. (B) Quantification of pull down of KLC1Myc in (A) relative to input; ${ }^{* * * *} \mathrm{p}<0.0001$ by unpaired two-tailed t test; $\mathrm{n}=3$; data represent mean \pm s.e.m. (C) Quantification of pull down of FLAG-KIF5A in (A) relative to input; ****p $<0.0001$ by unpaired two-tailed t test; $n=3$; data represent mean \pm s.e. $m$. (D) Schematic of the indicated constructs for KLC1; Heptad, Heptad repeat; TPR, tetratricopeptide repeat. (E) HEK 293T cells transfected with HA-SFPQ with empty vector, full length WT or tail-truncated Myc-KLC1. HA was IPed and blotted for Myc and HA. (F) Quantification of pull down in (E) relative to input; ${ }^{* *} p=0.0033$ by unpaired two-tailed $t$ test; $n=4$; data represent mean \pm s.e.m. (G) Schematic of the indicated constructs for KIF5A. (H) HEK 293T cell transfected with HA-SFPQ with empty vector, full length WT or tail-truncated FLAG-tagged KIF5A. HA was IPed and blotted for FLAG and HA. (I) Quantification of pull down in $(\mathrm{H})$ relative to input; ${ }^{* * * *} \mathrm{p}<0.0001$ by unpaired two-tailed $\mathrm{t}$ test; $\mathrm{n}=3$; data represent mean \pm s.e. $\mathrm{m}$.

Figure 3-figure supplement 1. KIF5A $\Delta$ Tail mutant binds to KLC1. 


\section{Figure 3-figure supplement 1. KIF5A $\triangle$ Tail mutant binds to KLC1.}

HEK 293 T cells transfected with KLC1-Myc and with either WT FLAG-tagged KIF5A or the $\triangle$ Tail mutant.

763

IP: KLC1-Myc

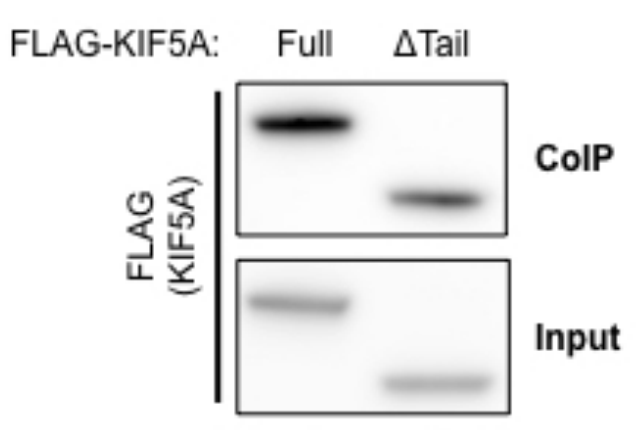

764 Myc was IPed and blotted for FLAG and Myc. 
A

B
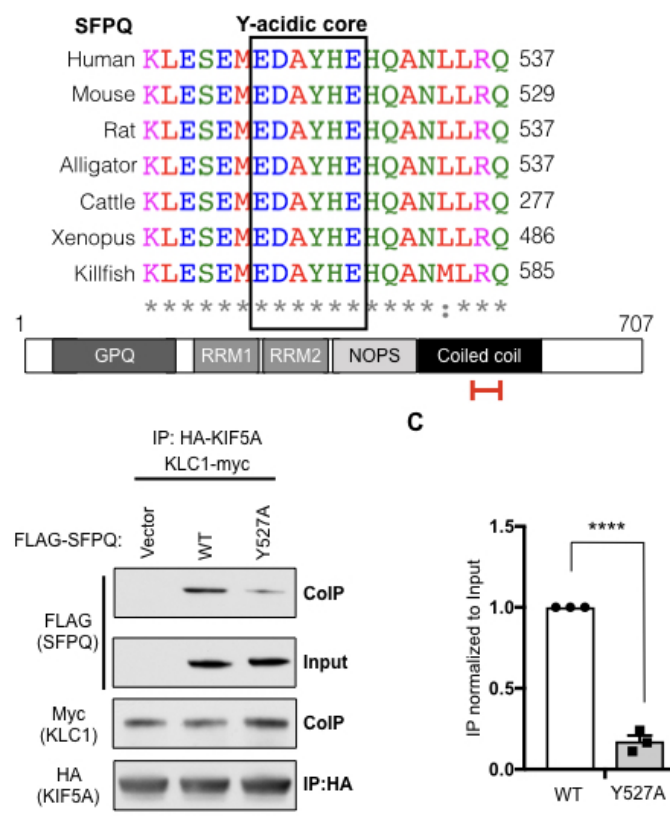

C

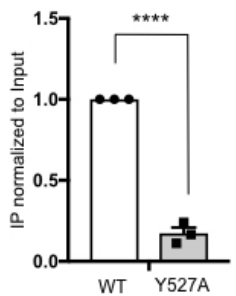

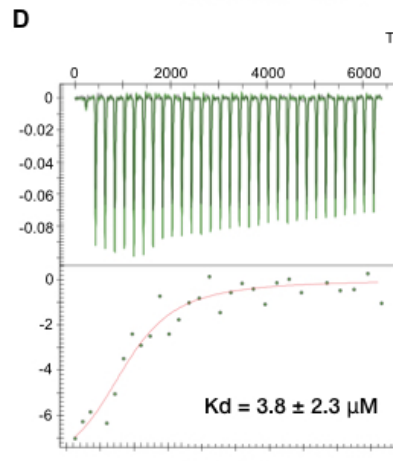

WT SFPQ

Time (sec)

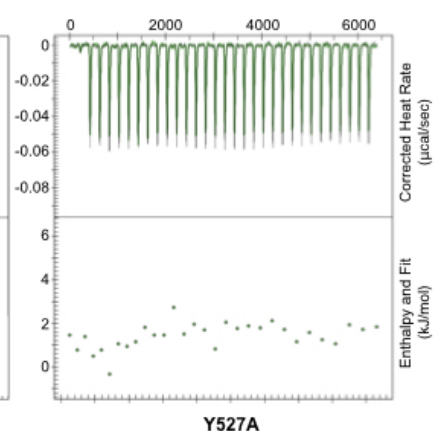

Figure 4. SFPQ directly binds to KLC1 through a Y-acidic motif within its coiled coil domain.

(A) Alignment of the sequence within the coiled coil domain of SFPQ containing the Y-acidic motif. On the bottom; schematic of the domains of SFPQ. Red bracket indicates the region containing the $Y$-acidic motif. GPQ, glycine proline glutamine-rich; RRM, RNA recognition motif; NOPS, NONA/paraspeckle domain. (B) HEK 293T cells transfected with HA-KIF5A, KLC1-Myc and with either empty vector, full length WT or Y527A FLAG-tagged SFPQ. HA was IPed and blotted for FLAG, Myc and HA. (C) Quantification of pull down in (B) relative to input; ${ }^{* * *} \mathrm{p}<0.0001$ by unpaired two-tailed $\mathrm{t}$ test; $\mathrm{n}=3$; data represent mean \pm s.e.m. (D) Isothermal titration calorimetry (ITC) measurements of the reference KLC1 (TPR1-6) fragment with either the WT peptide (ESEMEDAYHEHQANLLR) or the Y-acidic mutant, Y527A, peptide (ESEMEDAAHEHQANLLR) Of SFPQ.

Figure 4-figure supplement 1. SFPQ and JIP1 both share a Y-acidic motif. 


\section{Y-acidic core}

Human YTCPTEDIYLE 711

Mouse YTCPTEDIYLE 707

Rat YTCPTEDIYLE 708

Alligator YTCPTEDIYLE $700-(\mathrm{COOH})$

Rainbow Trout CSCPTEDIYLE 812

Roundworm FSHPTEDIYLE 361

Drosophila TAYPIEDIYIE 483

$: * * * *: *$

1

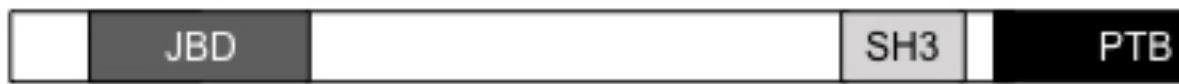

\section{Figure 4-figure supplement 1. SFPQ and JIP1 both share a Y-acidic motif.}

Alignment of the sequence within the C-terminal region of JIP1 containing the Y-acidic motif. On the bottom; schematic of the domains of JIP1. Red bracket indicates the region containing the Y-acidic motif. JBD, JNK binding domain; SH3, Src homology-3 domain; PTB, phosphotyrosine binding domain. 
bioRxiv preprint doi: https://doi.org/10.1101/2020.02.02.931204; this version posted February 3, 2020. The copyright holder for this preprint (which was not certified by peer review) is the author/funder, who has granted bioRxiv a license to display the preprint in perpetuity. It is made available under aCC-BY 4.0 International license.

785 Table 1. ITC parameters between KLC1 TPR1-6 fragment and WT SFPQ or Y527A Y-acidic mutant 786 peptide.

787

\begin{tabular}{|c|c|c|c|c|c|}
\hline $\begin{array}{c}\text { KLC1 } \\
\text { TPR1-6 } \\
\text { (Cell) }\end{array}$ & $\begin{array}{c}\text { SFPQ } \\
(\text { Syringe })\end{array}$ & $\mathbf{N}$ & $\begin{array}{c}\text { Kd } \\
(\mu \mathrm{M})\end{array}$ & $\begin{array}{c}\Delta \mathbf{H} \\
(\mathrm{kj} / \mathrm{mol})\end{array}$ & $\begin{array}{c}\Delta \mathbf{S} \\
(\mathrm{J} / \mathrm{mol} \cdot \mathrm{K})\end{array}$ \\
\cline { 2 - 6 } & WT & $0.964 \pm 0.143$ & $3.833 \pm 2.310$ & $-8.580 \pm 1.834$ & 74.92 \\
\cline { 2 - 6 } & Y527A & \multicolumn{4}{|c|}{ No Binding } \\
\hline
\end{tabular}

788

789

Table1-Source Data 1: Raw ITC data for WT SFPQ

790 Table1-Source Data 2: Raw ITC data for Y527A Y-acidic mutant 
791
A

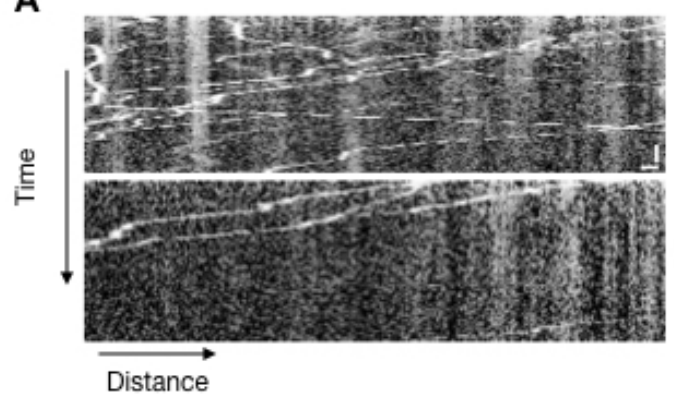

B

WT

Y527A

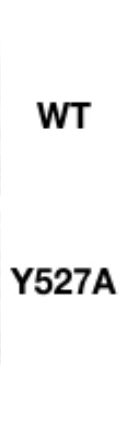

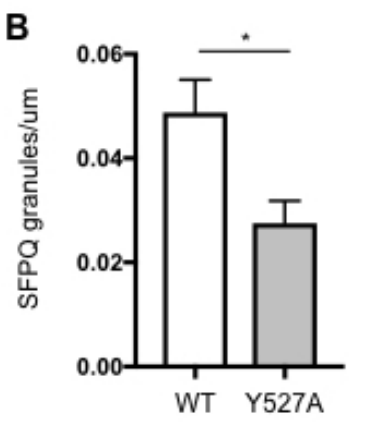

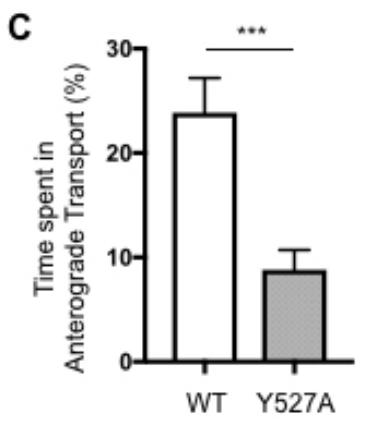

Figure 5. Direct binding of SFPQ to KIF5A/KLC1 is required for its transport in axons.

(A) Representative kymograph of WT and Y527A Halo-tagged SFPQ. Scale Bars: $2 \mu \mathrm{m}$ and $15 \mathrm{sec}$. (B) Average number of Halo-tagged WT and Y527A per micron of axon length. Analyzed from $n=25-33$ axons from two independent experiments; ${ }^{*} p=0.0117$; data represent mean \pm s.e.m. (C) Average percentage of time spent in anterograde transport for Halo-tagged WT and Y527A in axons of DRG sensory neurons. Analyzed from $n=25-33$ axons from 2 independent experiments; ${ }^{* *} \mathrm{p}=0.0005$; data represent mean \pm s.e.m.

Figure 5-figure supplement 1. SFPQ mode of binding to KLC1 is distinct from JIP1. 
A

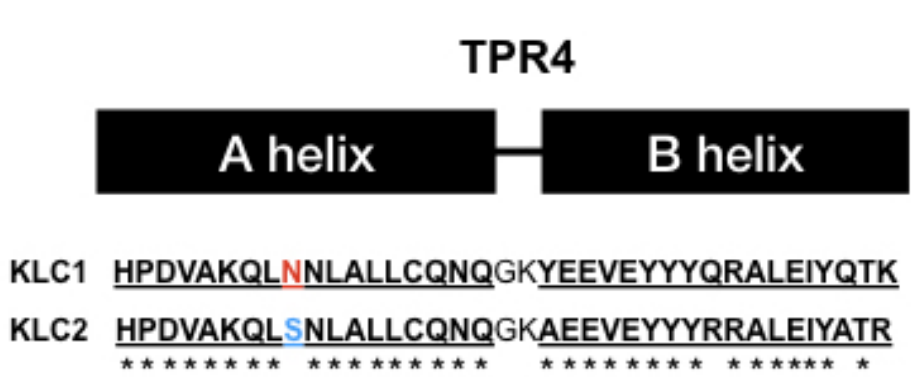

B

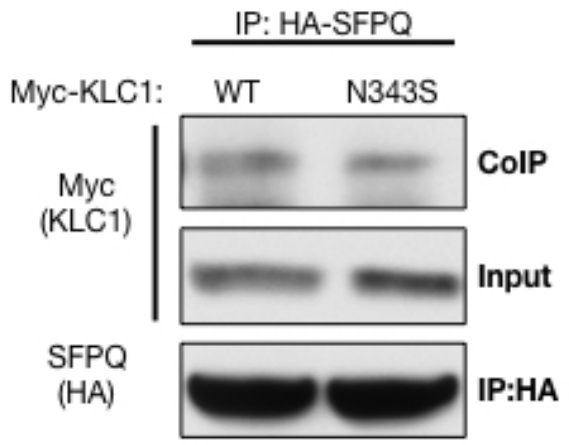

801

802

803

804

805

Figure 5-figure supplement 1. SFPQ mode of binding to KLC1 is distinct from JIP1.

(A) Schematic cartoon depicting the location of N343 on KLC1 in comparison to KLC2 on A helix of TPR4. (B) HEK 293T lysates transfected with HA-SFPQ, and with either Myc-tagged WT or N343S mutant of KLC1. HA-SFPQ was IPed and blotted against HA and Myc. 
A

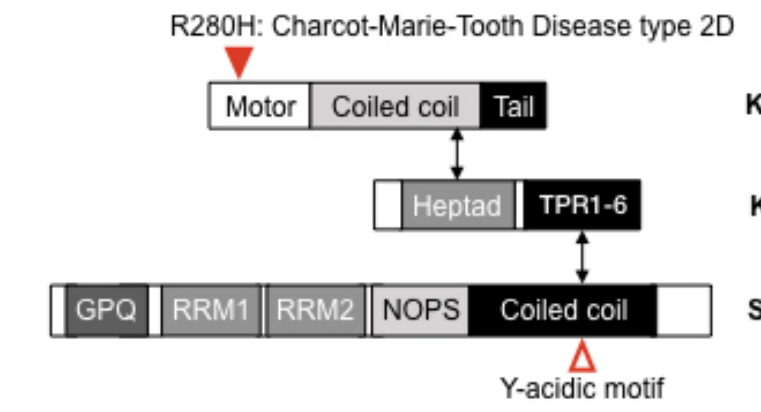

B

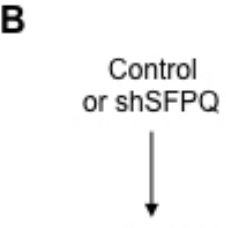

Rescue ShSFPQ with empty, WT, or Y-acid

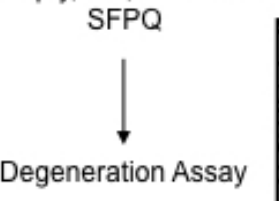

$\mathbf{E}$

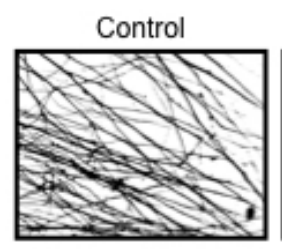

C
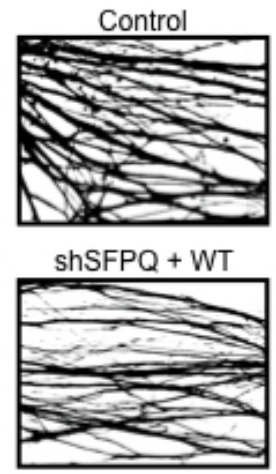

WT

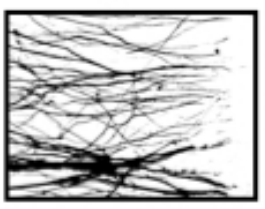

KIF5A

KLC1

SFPQ

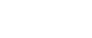

shSFPQ

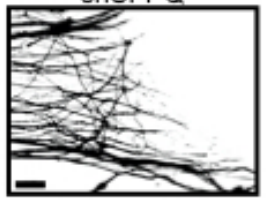

shSFPQ + Y-acid

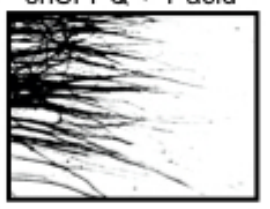

$\mathrm{R} 280 \mathrm{H}$

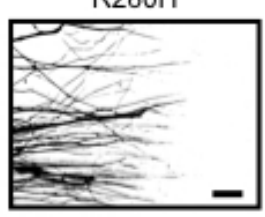

D

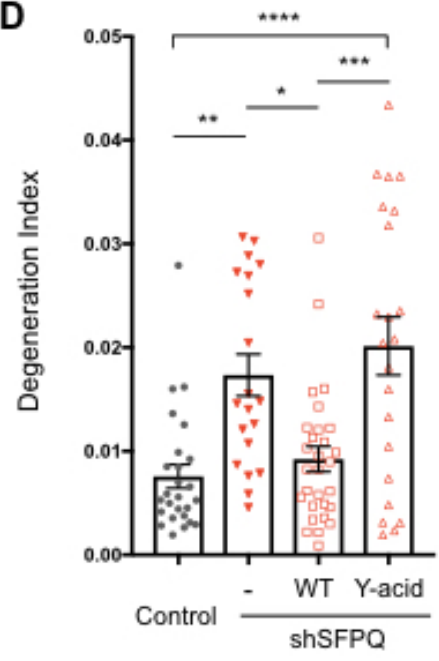

$\mathbf{F}$

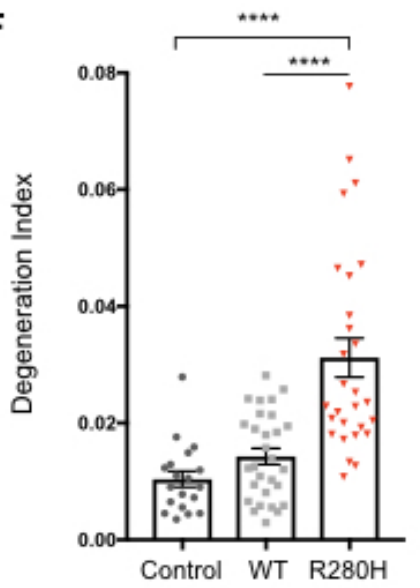

806

807

808

809

810

811

812

813

814

815

816

817

818

819

820

821

822

823

824

Figure 6. Defect in KIF5A-driven transport of SFPQ leads to axon degeneration in DRG sensory neurons.

(A) A schematic representation of KIF5A, KLC1 and SFPQ. Red closed arrowhead indicates the location of $\mathrm{R} 280 \mathrm{H}$ CMT2D mutation; and open arrowhead indicates the location of Y-acidic motif of SFPQ. (B) A flowchart of rescue experiment of degeneration assay using WT or the Y-acidic mutant of SFPQ. (C) Representative binarized Tuj1-labeled axons in compartmented cultures expressing control $(n=26)$ or shSFPQ rescued with empty vector $(n=20)$, WT $(n=29)$ or the Y-acidic mutant $(n=22)$ of SFPQ. From 3 independent experiments; Scale bar $100 \mu \mathrm{m}$. (D) Quantification of axon degeneration index of (C); ${ }^{*} \mathrm{p}=$ $0.0112, * * p=0.0019, * * * p=0.0002, * * * * p<0.0001$ by one way ANOVA; data represent mean \pm s.e.m. (E) Representative binarized Tuj1-labeled axons in compartmented cultures expressing control $(n=19)$, WT $(n=29)$ or R280H $(n=28)$ mutant of KIF5A. From 3 independent experiments; Scale bar $100 \mu m$. (F) Quantification of axon degeneration index of $(\mathrm{E}) ; * * * * \mathrm{p}<0.0001$ by one way ANOVA; data represent mean \pm s.e.m.

Figure 6-figure supplement 1. Expression of shRNA-resistant WT or Y-acidic GFP-tagged SFPQ. Figure 6-figure supplement 2. R280H mutation of KIF5A, which impairs transport, also reduces binding to SFPQ. 
bioRxiv preprint doi: https://doi.org/10.1101/2020.02.02 931204; this version posted February 3, 2020. The copyright holder for this preprint (which was not certified by peer review) is the author/funder, who has granted bioRxiv a license to display the preprint in perpetuity. It is made available under aCC-BY 4.0 International license.

825

826

827

828

829

830

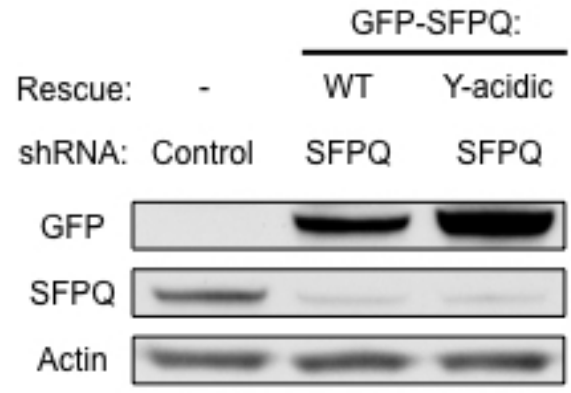

Figure 6-figure supplement 1. Expression of shRNA-resistant WT or Y-acidic GFP-tagged SFPQ. DRG neurons were infected with control or shRNA against SFPQ and rescued with empty, GFP-tagged WT or Y-acidic mutant of SFPQ. Protein lysates were blotted against GFP, endogenous SFPQ and actin as loading control. 
A

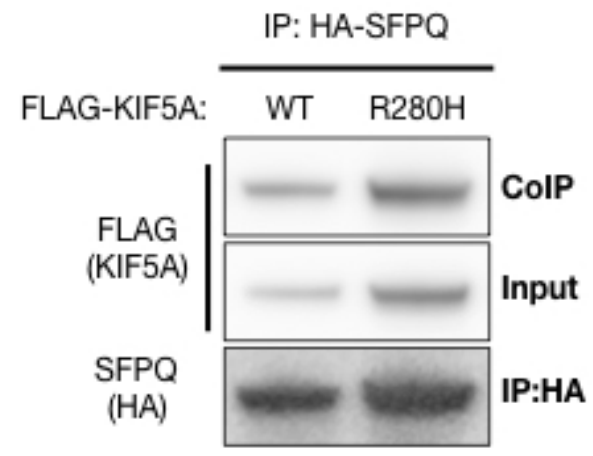

B

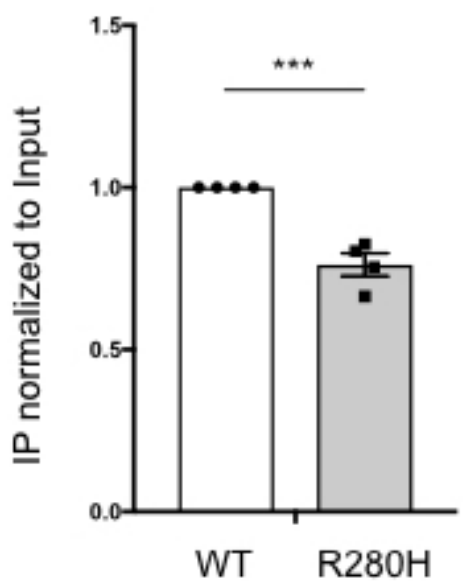

831

832

833

834

835

836
Figure 6-figure supplement 2. R280H mutation of KIF5A, which impairs transport, also reduces binding to SFPQ.

(A) HEK 293T lysates transfected with HA-SFPQ, and with either FLAG-tagged WT or R280H mutant of KIF5A. HA-SFPQ was IPed and blotted against FLAG and HA. (B) Quantification of pull down in (A) normalized to input. ${ }^{* * *} \mathrm{p}=0.0006$ by unpaired two-tailed $\mathrm{t}$ test; $\mathrm{n}=4$; data represent mean \pm s.e. $\mathrm{m}$. 
A

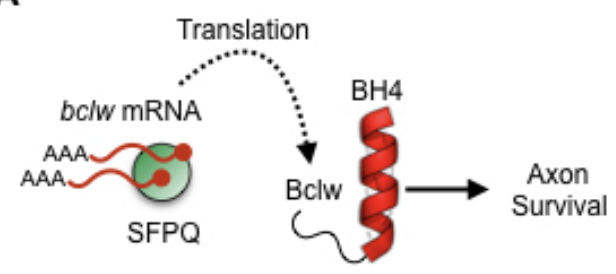

B
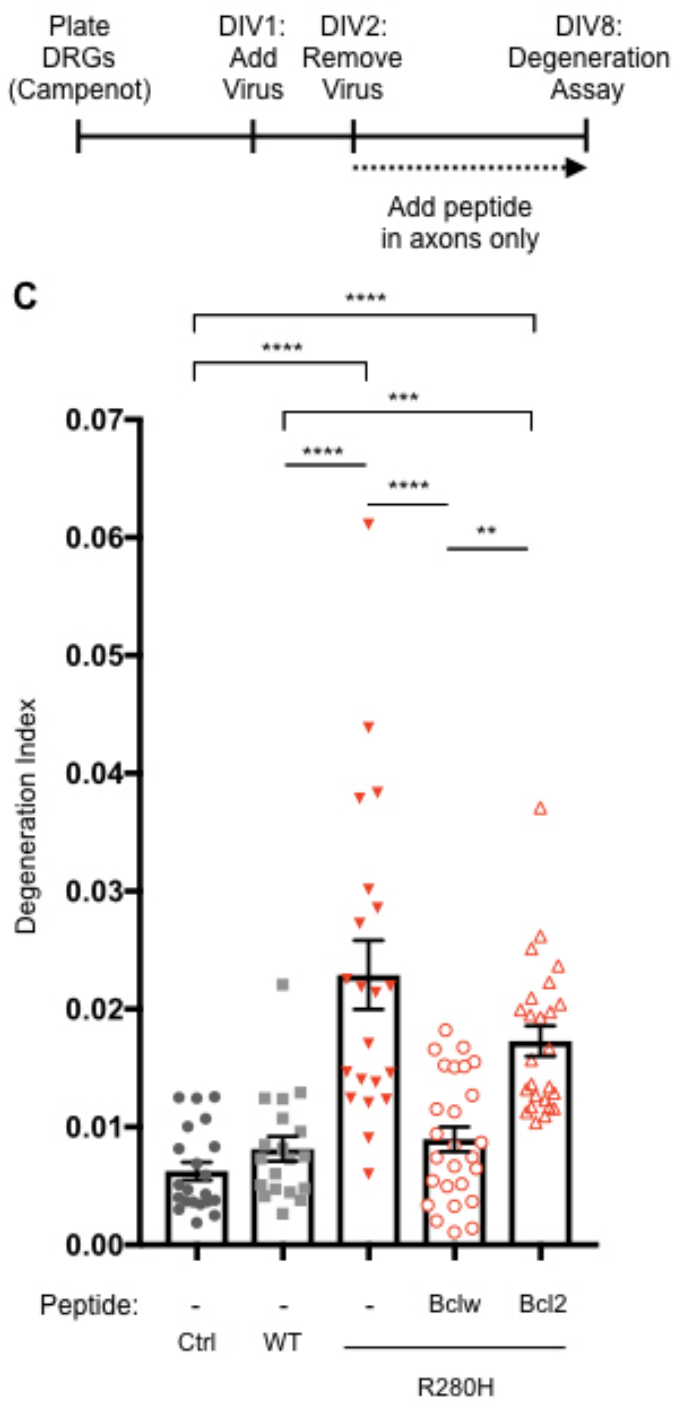

Figure 7. Axon degeneration caused by CMT2D R280H KIF5A mutation can be rescued by a Bclw mimetic peptide.

840 (A) A schematic of pathway for axon survival mediated by SFPQ. (B) Flowchart of Bclw peptide rescue experiment in DRG neurons cultured in compartmented Campenot chambers. (C) Quantification of axon degeneration index of control (Ctrl; $n=21)$, WT KIF5A $(n=19)$, R280H with either no peptide $(n=21)$, Bclw $(n=26)$, or $B c l 2$ peptide $(n=25)$. From 3 independent experiments; $* * p=0.0011,{ }^{* * *} p=0.0010, * * * * p$ 845 
846 Introduction-figure supplement 1. Bioinformatic analysis of SFPQ protein sequence.

847 Figure 1-figure supplement 1. Transport kinetics of SFPQ granules in axons of DRG sensory neurons.

848 Figure 2-figure supplement 1. Silver stain analysis of endogenous KLC1 and SFPQ IPs from DRGs and 849 verification of antibodies for KIF5A, KIF5B and KIF5C.

850 Figure 2-figure supplement 2. KIF5 motors differentially localizes to cell body and distal axons.

851 Figure 3-figure supplement 1. KIF5A $\triangle$ Tail mutant binds to KLC1.

852 Figure 4-figure supplement 1. SFPQ and JIP1 both share a Y-acidic motif.

853 Figure 5-figure supplement 1. SFPQ mode of binding to KLC1 is distinct from JIP1.

854 Figure 6-figure supplement 1. Expression of shRNA-resistant WT or Y-acidic GFP-tagged SFPQ.

855 Figure 6-figure supplement 2. R280H mutation of KIF5A, which impairs transport, also reduces binding

856 to SFPQ.

857

Table 1. ITC parameters between KLC1 TPR1-6 fragment and WT SFPQ or Y527A Y-acidic mutant

peptide.

860 Table1-Source Data 1: Raw ITC data for WT SFPQ

861 Table1-Source Data 2: Raw ITC data for Y527A Y-acidic mutant

Supplementary File 1: LC-MS/MS data

Video 1: Halo-SFPQ is transported in anterograde and retrograde manner in axons. Time-lapse movie was captured in axons of dorsal root ganglion sensory neurons grown in compartmented cultures. The

868 Video 2: Y527A mutation of SFPQ disrupts axonal transport of SFPQ. Time-lapse movie was captured in axons of dorsal root ganglion sensory neurons grown in compartmented cultures. The video was acquired every $1.5 \mathrm{sec}$ and played at $10 \mathrm{fps}$. 